\title{
Asymptotic behaviour of fast diffusions on graphs
}

\section{Adam Gregosiewicz ${ }^{1}$}

Received: 29 August 2019 / Accepted: 28 July 2020 / Published online: 30 September 2020

(c) The Author(s) 2020

\begin{abstract}
We study a diffusion process on a finite graph with semipermeable membranes on vertices. We prove, in $L^{1}$ and $L^{2}$-type spaces that for a large class of boundary conditions, describing communication between the edges of the graph, the process is governed by a strongly continuous semigroup of operators, and we describe asymptotic behaviour of the diffusion semigroup as the diffusions' speed increases at the same rate as the membranes' permeability decreases. Such a process, in which communication is based on the Fick law, was studied by Bobrowski (Ann. Henri Poincare 13(6):1501-1510, 2012) in the space of continuous functions on the graph. His results were generalized by Banasiak et al. (Semigroup Forum 93(3):427-443, 2016). We improve, in a way that cannot be obtained using a very general tool developed recently by Engel and Kramar Fijavž (Evolut. Equ. Control Theory 8(3)3:633$661,2019)$, the results of J. Banasiak et al.
\end{abstract}

Keywords Diffusion on graphs · Transmission conditions · Asymptotic behaviour . Singular perturbations $\cdot$ Semigroups of operators $\cdot$ Sesquilinear forms

\section{Introduction}

A number of papers on evolution operators acting on graphs have been published in the last decades, see for example [8, 20] and references given there. Here we consider a finite graph $\mathcal{G}$ without loops, and we assume that there is a Markov process on $\mathcal{G}$, which on each edge behaves like a Brownian motion with given variance. Moreover, we suppose that each vertex is a semipermeable membrane with given permeability coefficients, that is for each vertex there are nonnegative numbers $p_{i j}$, describing the possibility of a particle passing through the membrane from the $i$-th to the $j$-th edge.

Communicated by Jerome A. Goldstein.

Adam Gregosiewicz

a.gregosiewicz@pollub.pl

1 Lublin University of Technology, ul. Nadbystrzycka 38A, 20-618 Lublin, Poland 
Such processes were used to model synaptic depression dynamic, see [7] for details, and were studied by Bobrowski [4] with the underlying space being the space of continuous functions on $\mathcal{G}$. He proved that these processes are governed by strongly continuous semigroups, and then, moreover, if the diffusion's speed increases to infinity with the same rate as permeability coefficients decreases to zero, that there is a limit process which behaves like a Markov chain on the vertices of the line graph of $\mathcal{G}$. Because the analysis takes place in the space of continuous functions, the related semigroups describe dynamics of (weighted) conditional expected values of these processes.

The first aim of this paper is to prove analogous generation and asymptotic results but in a different space, in order to obtain the dynamics of densities of the processes' distributions. We consider a "dual" description of such processes with the underlying space being $L^{1}$-type space of Lebesgue integrable functions on $\mathcal{G}$. One may wish to mimic the argument of the continuous case but this is not fully possible, thus a different method is needed. We use a modification of Nickel's approach [22] to Greiner's perturbation [14], and we give a new and short proof of the generation theorem in the dual case obtained previously in [13]. Next, via Kurtz's convergence theorem [10, Theorem 1.7.6], we describe asymptotics of the related semigroups.

The methods described above allow us to prove generation and asymptotic theorems for diffusion semigroups on graphs for a large class of boundary conditions. In particular, we improve results obtained by Banasiak et al. in [2, 3]. Recently Engel and Kramar Fijavž studied [8] such problems in a very general setting, however, to the best of our knowledge, our main theorem cannot be derived from [8, Theorem 2.3].

Finally, in the last part of the paper, we show that in a Hilbert space setting, specifically in the space of square integrable functions on $\mathcal{G}$, we can approach the problem via classical theory of sesquilinear forms. Here our main tool is Ouhabaz's monotone convergence theorem [23, Theorem 5], which generalizes Simon's theorem [27, Theorem 3.1].

\subsection{Continuous case}

As in [4], let $\mathcal{G}=(\mathcal{V}, \mathcal{E})$ be a finite graph without loops, where $\mathcal{V} \subset \mathbb{R}^{3}$ is the set of vertices, and $\mathcal{E}$ is the set of edges of finite length. The edges are seen as $C^{1}$ curves connecting vertices. Let $N$ be the number of edges and denote

$$
\mathcal{N}:=\{1, \ldots, N\}, \quad \mathbb{N}:=\{1,2, \ldots\} .
$$

For each $i \in \mathcal{N}$, by convention, we call the initial and terminal vertices of the $i$-th edge $E_{i}$ its "left" and "right" endpoints. We denote them by $L_{i}$ and $R_{i}$, respectively. Let

$$
S=\biguplus_{i \in \mathcal{N}} E_{i}
$$

be the disjoint union of the edges. Note that there can be many "copies" of the same vertex in $S$, treated as endpoints of different edges. Then $S$ is a disconnected compact topological space, and we denote by $C(S)$ the space of continuous functions 
on $S$ with standard supremum norm. The space is isometrically isomorphic to $C\left(E_{1}\right) \times \cdots \times C\left(E_{N}\right)$, where $C\left(E_{i}\right)$ is the space of continuous functions on the edge $E_{i}$. The latter space is isometrically isomorphic to the space $C\left[0, d_{i}\right]$ of continuous functions defined on the interval $\left[0, d_{i}\right]$, where $d_{i}$ is the length of the $i$-th edge. Therefore we may identify $f \in C(S)$ with $\left(f_{i}\right)_{i \in \mathcal{N}}$, where $f_{i}$ is a member of $C\left(E_{i}\right)$. Whenever possible, we consider $f \in C(S)$ as a single function on a disconnected space $S$, and use the edgewise identification $f=\left(f_{i}\right)_{i \in \mathcal{N}}$ when needed. Note that it makes sense to speak about differentiable functions on $S$, and in particular, by $C^{k}(S)$ we denote the space of all $k$-times continuously differentiable functions on $S$.

For each $i \in \mathcal{N}$, let $l_{i}$ and $r_{i}$ be nonnegative real numbers giving the rates at which particles pass through the membrane from the $i$-th edge to the edges incident in the left and right endpoints, respectively. Also, for $i, j \in \mathcal{N}$ such that $i \neq j$ let $l_{i j}$ and $r_{i j}$ be nonnegative real numbers satisfying $\sum_{j \neq i} l_{i j} \leqslant l_{i}$ and $\sum_{j \neq i} r_{i j} \leqslant r_{i}$; the summation here is taken over all $j \in \mathcal{N}$ such that $j \neq i$. These numbers determine the probability that after filtering through the membrane at $L_{i}$, respectively $R_{i}$, from the $i$-th edge, a particle will enter the $j$-th edge. More specifically, the probability that a particle after filtering through the membrane at $L_{i}$, respectively $R_{i}$, will enter the $j$-th edge equals $l_{i j} / l_{i}$, respectively $r_{i j} / r_{i}$; see [4] for a more detailed description. By default, if $E_{j}$ is not incident with $L_{i}$, respectively $R_{i}$, we put $l_{i j}=0$, respectively $r_{i j}=0$. For $i \neq j$ we denote by $L_{i j}$ and $R_{i j}$, the left, respectively right, endpoint of $E_{i}$ seen as an endpoint of $E_{j}$; if $E_{i}$ and $E_{j}$ are not adjacent, then we leave $L_{i j}$ and $R_{i j}$ undefined. If, for example, $E_{j}$ is incident to the left endpoint of $E_{i}$, then $L_{i j}$ equals $L_{j}$ or $R_{j}$.

With these notations, we introduce the following transmission conditions. Let $\left(\kappa_{n}\right)_{n \in \mathbb{N}}$ be a nondecreasing sequence of real numbers satisfying

$$
\kappa_{n} \geq 1
$$

that diverge to infinity. For a fixed $n \in \mathbb{N}$ we consider functions $f \in C(S)$ satisfying

$$
\kappa_{n} f^{\prime}\left(L_{i}\right)=l_{i} f\left(L_{i}\right)-\sum_{j \neq i} l_{i j} f\left(L_{i j}\right), \quad i \in \mathcal{N},
$$

where $f^{\prime}\left(L_{i}\right)$ is the right-hand derivative of $f$ at $L_{i}$, and

$$
-\kappa_{n} f^{\prime}\left(R_{i}\right)=r_{j} f\left(R_{i}\right)-\sum_{j \neq i} r_{i j} f\left(R_{i j}\right), \quad i \in \mathcal{N},
$$

where $f^{\prime}\left(R_{i}\right)$ is the left-hand derivative of $f$ at $R_{i}$. By convention, if $L_{i j}$ or $R_{i j}$ is not defined (in the case when $E_{i}$ and $E_{j}$ are not adjacent in $L_{i}$ or $R_{i}$ ) we let $f\left(L_{i j}\right)=0$, respectively $f\left(R_{i j}\right)=0$. Recall also that the space $S$ is disconnected, and even if, for example, $L_{i}$ is the same vertex of the graph as $R_{j}$, they differ as elements of $S$, and consequently $f\left(L_{i}\right)$ may differ from $f\left(R_{j}\right)$.

Let $\sigma \in C(S)$ be a continuous positive function defined on $S$, which on each edge is constant, that is for each $i \in \mathcal{N}$ we have

$$
\sigma(x)=\sigma_{i}, \quad x \in E_{i},
$$

where $\sigma_{i}$ is a positive real number. We define the operator $A_{n}$ in $C(S)$ by 


$$
A_{n} f=\kappa_{n} \sigma f^{\prime \prime}, \quad f \in D\left(A_{n}\right),
$$

where $\sigma f^{\prime \prime}$ is the product of $\sigma$ and $f^{\prime \prime}$, that is the function $x \mapsto \sigma(x) f^{\prime \prime}(x)$, and

$$
D\left(A_{n}\right):=\left\{f \in C^{2}(S): \text { f satisfies (1.1) and (1.2) }\right\},
$$

that is $f \in D\left(A_{n}\right)$ if and only if $f$ is a twice continuously differentiable function on $S$ satisfying transmission conditions (1.1)-(1.2). Note that if $f$ is identified with $\left(f_{i}\right)_{i \in \mathcal{N}}$, then we may identify $A_{n} f$ with $\left(\kappa_{n} \sigma_{i} f_{i}^{\prime \prime}\right)_{i \in \mathcal{N}}$, however we feel that it is more natural to consider $f$ and $A_{n} f$ as continuous real functions on the (disconnected) space $S$, as in [4], and write

$$
A_{n} f(x)=\left(\kappa_{n} \sigma f^{\prime \prime}\right)(x)=\kappa_{n} \sigma(x) f^{\prime \prime}(x)
$$

for all $x \in S$.

Before stating the main result of [4], we recall the notion of Feller semigroups; see for example [10, Section 4.2] or [18, Chapter 3]. Let 4, Proposition 2.1 and Theorem 2.2) For every $\Omega$ be a compact topological space and $C(\Omega)$ be the space of all continuous functions on $\Omega$. The space $C(\Omega)$ with the standard supremum norm is a Banach space. We say that a strongly continuous semigroup $\left\{\mathrm{e}^{t A}\right\}_{t \geqslant 0}$ in $C(\Omega)$ is a Feller semigroup if and only if for all $t \geq 0$ the operator $\mathrm{e}^{t A}$ is a nonnegative contraction, that is $\left\|\mathrm{e}^{t A}\right\|_{\mathcal{L}(C(\Omega))} \leqslant 1$, and $\mathrm{e}^{t A} f \geqslant 0$, provided that $f \in C(\Omega)$ is nonnegative. Here, $\|\cdot\|_{\mathcal{L}(C(\Omega))}$ is the operator norm related to the standard supremum norm in $C(\Omega)$. Moreover, we say that the semigroup is conservative if $\mathrm{e}^{t A} \mathbb{1}_{\Omega}=\mathbb{1}_{\Omega}$, where $\mathbb{1}_{\Omega}$ is the constant function that equals 1 on $\Omega$.

Theorem 1.1 ( $n \in \mathbb{N}$ the operator $A_{n}$ given by (1.3) generates a Feller semigroup in $C(S)$. The semigroup is conservative if and only if $\sum_{j \neq i} l_{i j}=l_{i}$ and $\sum_{j \neq i} r_{i j}=r_{i}$ for all $i \in \mathcal{N}$.

Moreover, for every $f \in C(S)$ and $t>0$ it follows that

$$
\lim _{n \rightarrow+\infty} \mathrm{e}^{t A_{n}} f=\mathrm{e}^{t Q} P f
$$

in $C(S)$, where $P$ is the projection of $C(S)$ onto the space $C_{0}(S)$ of functions that are constant on each edge, given by $\mathrm{Pf}=\left(d_{i}^{-1} \int_{E_{i}} f\right)_{i \in \mathcal{N}}$, while $Q$ is the operator in $C_{0}(S)$ which may be identified with the matrix $\left(q_{i j}\right)_{i, j \in \mathcal{N}}$ with

$$
q_{i j}= \begin{cases}\sigma_{i} d_{i}^{-1}\left(l_{i j}+r_{i j}\right), & i \neq j, \\ -\sigma_{i} d_{i}^{-1}\left(l_{i}+r_{i}\right), & i=j .\end{cases}
$$

The convergence here is uniform on compact subsets of $(0, \infty)$. For $f \in C_{0}(S)$, the formula holds also for $t=0$, and the convergence is uniform on compact subsets of $[0, \infty)$.

For the Feller semigroup $\left\{\mathrm{e}^{t A_{n}}\right\}_{t \geq 0}$ there is a Feller process $X_{n}(t), t \geq 0$ on the graph $\mathcal{G}$ satisfying 


$$
\mathrm{e}^{t A_{n}} f(x)=E^{x} f\left(X_{n}(t)\right), \quad x \in S, t \geq 0, f \in C(S) ;
$$

see [18, Theorem 3.26]. Here $E^{x}$ is the expectation conditional on the process starting at $x$. The process $X_{n}(t)$ on each edge $E_{i}$ behaves like a Brownian motion with variance $\kappa_{n} \sigma_{i}$. When a particle that moves inside $E_{i}$ hits, for example, $L_{i}$, then is bounces from the membrane. The time that this particle spends at the membrane may be measured by the Lévy local time $t^{+}$, see [7, Section 4] and the references given there. After a random time $T$ distributed according to

$$
P(T>t)=\mathrm{e}^{-l_{i} t^{+}}, \quad t \geq 0,
$$

the particle pass through the membrane at $L_{i}$, and the probability that it will filter to $E_{j}$ equals $l_{i j} / l_{i}$. There is also a possibility that after filtering through the membrane the particle is removed, with probability $1-\sum_{j \neq i} l_{i j} / l_{i}$, from the state space. We stress that in this model there can be many copies of a vertex of the graph $\mathcal{G}$ in $S$, and consequently the behaviour of a particle hitting a membrane depends on the "side" of the vertex that this particle hit.

Therefore Theorem 1.1 describes the dynamics of the conditional expected value of the position of a particle which behaves according to the process $X_{n}(t)$ as the diffusions' speed increase to infinity with the same rate as permeability coefficients decrease to zero.

\subsection{Main results}

The first aim of this paper is to prove a "dual" version of Theorem 1.1. We define a semigroup in $L^{1}$-type space that is related to the process $X_{n}(t)$ introduced in Sect. 1.1. In contradistinction to (1.4), the semigroup describes the dynamics of densities of the process; see [17, Section 7.10]. Loosely speaking, the result is as follows; for the precise formulation see Theorem 2.3 and Corollary 2.17.

Recall, see for example [17], that a strongly continuous semigroup $\{T(t)\}_{t \geq 0}$ in $L^{1}$-type space $\mathbb{X}$ is called Markov, if it is positive and preserves the norm of nonnegative functions, that is for all $f \in \mathbb{X}, f \geq 0$, it follows that $T(t) f \geq 0$ and $\|T(t) f\|_{X}=\|f\|_{X}$ for every $t \geq 0$. If we replace the last condition by $\|T(t) f\|_{\mathbb{X}} \leq\|f\|_{\mathbb{X}}$, then we call $\{T(t)\}_{t \geq 0}$ a sub-Markov semigroup.

Dual Theorem For the semigroup generated by $A_{n}$, let $\left\{T_{n}(t)\right\}_{t \geq 0}$ be the adjoint semigroup in the space of finite signed regular Borel measures on $S$. Then the space of Lebesgue integrable functions on $S$ is invariant for $\left\{T_{n}(t)\right\}_{t \geq 0}$, and the restriction $\left\{T_{n}(t)_{\mid}\right\}_{t \geq 0}$ of $\left\{T_{n}(t)\right\}_{t \geq 0}$ to that space is a sub-Markov semigroup. Moreover, the semigroup is Markov, provided that the semigroup generated by $A_{n}$ is conservative.

Furthermore, as $n$ goes to infinity the restricted semigroups $\left\{T_{n}(t)_{\mid}\right\}_{t \geq 0}$ converge strongly to $\mathrm{e}^{t} Q P$ for the projection $P$ and operator $Q$ given by the same formulas as in Theorem 1.1 concerning $C(S)$ case. 
Notice that, by the first part of the Dual Theorem, for every finite signed regular Borel measure $v$ on $S$, the measure $T_{n}(t) v, t \geq 0$ is absolutely continuous with respect to the Lebesgue measure, provided that so is $v$.

We prove that the generators of the semigroups $\left\{T_{n}(t)_{\mid}\right\}_{t \geq 0}$ are diffusion operators that are rescaled versions of the operator $A$ defined in the space of Lebesgue integrable functions on $S$ by

$$
A \varphi=\sigma \varphi^{\prime \prime},
$$

with the domain composed of functions satisfying boundary conditions of the form

$$
\varphi^{\prime}\left(L_{i}\right)=F_{L, i} \varphi, \quad \varphi^{\prime}\left(R_{i}\right)=F_{R, i} \varphi, \quad i \in \mathcal{N} .
$$

In our setting $F_{L, i}$ and $F_{R, i}$ are a specific boundary functionals that are in a sense dual to the right-hand sides of (1.1)-(1.2). One can of course consider the operator $A$ given by (1.5) for a generalized boundary functionals. For example, in [3] the authors studied the problem for functionals that are related to sums of Dirac measures concentrated at vertices. In particular, the fact that $A_{n}^{*}$ 's are generators is a special case of [3, Theorem 2.4]. However, in the Dual Theorem, we are interested not only in the fact that these operators with specific boundary conditions generate semigroups - the relation to the semigroups introduced in [4] is important to us. This allows interpreting the Dual Theorem in probabilistic terms.

Nevertheless, it turns out that we can extend the method used in the proof of the Dual Theorem to obtain a generation result for diffusion operators with much more general boundary conditions. Specifically, we prove the following; see Corollary 2.7.

Main Generation Theorem The operator A given by (1.5) generates a strongly continuous semigroup in the space of Lebesgue integrable functions on $S$, provided that $F_{L, i}$ and $F_{R, i}$ are bounded functionals on the first order Sobolev-type space of functions on $S$.

Additionally, for $\kappa_{n} \geq 1$ as in Sect. 1.1, introducing $A_{n}$ as a singular perturbation of $A$, that is

$$
A_{n} \varphi=\kappa_{n} \sigma \varphi^{\prime \prime}
$$

with domain as for $A$ with $F_{L, i}$ and $F_{R, i}$ replaced by $\kappa_{n}^{-1} F_{L, i}$ and $\kappa_{n}^{-1} F_{R, i}$, we also describe the asymptotic behaviour of semigroups generated by $A_{n}$ 's under mild assumptions on $F_{L, i}$ and $F_{R, i}$; see Theorem 2.11.

Main Asymptotics Theorem As $n$ goes to infinity the semigroups generated by $A_{n}$ 's converge strongly to $\mathrm{e}^{t Q} P$ for the projection $P$ given by the same formula as in Theorem 1.1, and an operator $Q$, see (2.32), defined in the space of functions on $S$ that are constant on each edge.

Both main theorems generalize results obtained in [2, 3]. We also note that the Main Generation Theorem cannot be derived from [8, Theorem 2.3] - a recent generation 
result concerning diffusion (and waves) operators on graphs for a large class boundary conditions.

Finally, see Theorems 3.2 and 3.6, we also consider the problem in $L^{2}$-type Hilbert space setting. Here it is possible to use the classical approach via sesquilinear forms to prove a generation theorem, and in order to check the asymptotics of the related semigroups, we use methods introduced by Simon [27] and Ouhabaz [23]. Comparing to the $L^{1}$-type case, in the Hilbert space setting proofs are easier, and rather straightforward. However, it is not clear whether the results say anything about the underlying stochastic process.

\section{Analysis in $L^{1}(S)$}

We consider a model that is in a way dual to that described in Sect. 1.1, by investigating the restriction of the adjoint of $A_{n}$ to an $L^{1}$-type space.

In order to set up notations, we fix

$$
\left\|\left(x_{i}\right)_{i=1, \ldots, k}\right\|_{\mathbb{R}^{k}}:=\sum_{i=1}^{k}\left|x_{i}\right|, \quad\left(x_{i}\right)_{i=1, \ldots, k} \in \mathbb{R}^{k}
$$

to be the norm in the Euclidean space $\mathbb{R}^{k}$. Next, for an interval $I \subset \mathbb{R}$ equipped with the Lebesgue measure, let $L^{1}(I)$ be the real space of (equivalence classes of) Lebesgue integrable real functions defined on $I$. By $\|\cdot\|_{L^{1}(I)}$ we denote the standard norm

$$
\|\varphi\|_{L^{1}(I)}:=\int_{I}|\varphi(t)| \mathrm{d} t, \quad \varphi \in L^{1}(I) .
$$

Moreover, let $W^{k, 1}(I)$ be the $k$-th order Sobolev subspace of $L^{1}(I)$, that is the space of functions $\varphi \in L^{1}(I)$ that are $k$-times weakly differentiable and such that $\varphi^{(k)} \in L^{1}(I)$. In particular, the space $W^{1,1}(I)$ with the norm

$$
\|\varphi\|_{W^{1,1}(I)}:=\|\varphi\|_{L^{1}(I)}+\left\|\varphi^{\prime}\right\|_{L^{1}(I)}, \quad \varphi \in W^{1,1}(I)
$$

is a Banach space, and by the Sobolev embedding theorem for each $\varphi \in W^{1,1}(I)$ there exists a continuous representant $\tilde{\varphi}$ of $\varphi$, and moreover $\|\tilde{\varphi}\|_{C(I)} \leq C\|\varphi\|_{W^{1,1}(S)}$ for some constant $C>0$ that does not depend on $\varphi$. Finally, if $\left(\mathbb{X},\|\cdot\|_{\mathbb{X}}\right)$ and $\left(\mathbb{Y},\|\cdot\|_{\mathbb{Y}}\right)$ are Banach spaces, then by $\mathcal{L}(\mathbb{X}, \mathbb{Y})$ we denote the space of bounded linear operators from $\mathbb{X}$ to $\mathbb{Y}$ equipped with the standard operator norm $\|\cdot\|_{\mathcal{L}(\mathbb{X}, \mathbb{Y})}$. If $\mathbb{X}=\mathbb{Y}$, then we just write $\mathcal{L}(\mathbb{X})$. Also, by $I_{\mathbb{X}}$ we denote the identity operator in $\mathbb{X}$.

\subsection{Adjoint of the operator $A_{n}$}

Using the same identification as in Sect. 1.1, we consider the space

$$
L^{1}(S):=\left\{\varphi: \varphi_{\mid E_{i}} \in L^{1}\left(E_{i}\right), i \in \mathcal{N}\right\}
$$


Here $L^{1}\left(E_{i}\right)$ is the space of (equivalence classes of) Lebesgue integrable functions on $E_{i}$, identified with $L^{1}\left(0, d_{i}\right)$. More precisely, if $\ell_{i}(t)$ is the unique point on the edge $E_{i}$, whose distance from $L_{i}$ (along the edge) is $t \in\left(0, d_{i}\right)$, then a function $\varphi \in L^{1}\left(E_{i}\right)$ is identified with $\varphi \circ \ell_{i} \in L^{1}\left(0, d_{i}\right)$. Such an identification is an isometric isomorphism. Now, if we equip $S$ with the Lebesgue measure $m$, then for every $\varphi \in L^{1}(S)$ we have

$$
\int_{S} \varphi(x) m(\mathrm{~d} x)=\sum_{i \in \mathcal{N}} \int_{E_{i}} \varphi(x) m(\mathrm{~d} x)=\sum_{i \in \mathcal{N}} \int_{0}^{d_{i}} \varphi \circ \ell_{i}(t) \mathrm{d} t .
$$

We introduce the norm $\|\cdot\|_{L^{1}(S)}$ in $L^{1}(S)$ by

$$
\|\varphi\|_{L^{1}(S)}:=\int_{S}|\varphi| \mathrm{d} m, \quad \varphi \in L^{1}(S),
$$

and note that $L^{1}(S)$ equipped with $\|\cdot\|_{L^{1}(S)}$ is a Banach space. As in Sect. 1.1, we will interchangeably consider $\varphi \in L^{1}(S)$ as a single function defined on the disconnected space $S$, or as a vector $\varphi=\left(\varphi_{i}\right)_{i \in \mathcal{N}}$, where $\varphi_{i} \in L^{1}\left(E_{i}\right)$. Furthermore, let $W^{k, 1}(S)$ be the Sobolev-type space on $S$, that is the subspace of $L^{1}(S)$ composed of (equivalence classes of) functions $\varphi \in L^{1}(S)$ such that $\varphi_{\mid E_{i}} \in W^{k, 1}\left(E_{i}\right)$ for all $i \in \mathcal{N}$. Note that, in particular, $W^{1,1}(S)$ with the norm

$$
\|\varphi\|_{W^{1,1}(S)}:=\sum_{i \in \mathcal{N}}\left\|\varphi_{\mid E_{i}}\right\|_{W^{1,1}\left(E_{i}\right)}, \quad \varphi \in W^{1,1}(S)
$$

is a Banach space, and moreover we can view each $\varphi \in W^{1,1}(S)$ as a continuous function.

Let $\sigma,\left(\kappa_{n}\right)_{n \in \mathbb{N}}$, and all $l_{i}, r_{i}, l_{i j}, r_{i j}$ 's be as in Sect. 1.1. For each $n \in \mathbb{N}$ and each $i \in \mathcal{N}$ let us consider for $\varphi \in W^{2,1}(S)$ the transmission conditions

$$
\begin{aligned}
& \kappa_{n} \sigma_{i} \varphi^{\prime}\left(L_{i}\right)=\sigma_{i} l_{i} \varphi\left(L_{i}\right)-\sum_{j \in I_{i}^{L}}\left[\sigma_{j} l_{j i} \varphi\left(L_{j}\right)+\sigma_{j} r_{j i} \varphi\left(R_{j}\right)\right], \\
& \kappa_{n} \sigma_{i} \varphi^{\prime}\left(R_{i}\right)=\sum_{j \in I_{i}^{R}}\left[\sigma_{j} l_{j i} \varphi\left(L_{j}\right)+\sigma_{j} r_{j i} \varphi\left(R_{j}\right)\right]-\sigma_{i} r_{i} \varphi\left(R_{i}\right) ;
\end{aligned}
$$

here, $I_{i}^{L}$ and $I_{i}^{R}$ are the sets of indices $j \neq i$ of edges incident in $L_{i}$ and $R_{i}$, respectively. Notice that, since there are no loops, for a given $i \in \mathcal{N}$ at least one of $l_{j i}, r_{j i}$ is zero, and hence at most one of the terms $\sigma_{j} l_{j i} \varphi\left(L_{j}\right)$ and $\sigma_{j} r_{j i} \varphi\left(R_{j}\right)$ is taken into account. Denoting the right-hand sides of (2.1) and (2.2) by, respectively, $\sigma_{i} F_{L, i} \varphi$ and $\sigma_{i} F_{R, i} \varphi$, we may rewrite these conditions in the form

$$
\kappa_{n} \varphi^{\prime}\left(L_{i}\right)=F_{L, i} \varphi, \quad \kappa_{n} \varphi^{\prime}\left(R_{i}\right)=F_{R, i} \varphi, \quad i \in \mathcal{N},
$$

and consider $F_{L, i}, F_{R, i}$ as linear functionals in $W^{2,1}(S)$. With this setup, for each $n \in \mathbb{N}$ we define the operator $A_{n}^{*}$ in $L^{1}(S)$ by 


$$
A_{n}^{*} \varphi:=\kappa_{n} \sigma \varphi^{\prime \prime}, \quad \varphi \in D\left(A_{n}^{*}\right)
$$

with domain

$$
D\left(A_{n}^{*}\right):=\left\{\varphi \in W^{2,1}(S): \varphi \text { satisfies }(2.3)\right\} .
$$

Now we establish the relation between $A_{n}$ from Sect. 1.1 and $A_{n}^{*}$ defined above. Let $M(S)$ be the dual space of $C(S)$. By the Riesz representation theorem, see [11, Corollary 7.18], $M(S)$ is the space of finite signed regular Borel measures on $S$. With the semigroup $\left\{\mathrm{e}^{t A_{n}}\right\}_{t \geq 0}$ in $C(S)$ we can associate the adjoint semigroup $\{T(t)\}_{t \geq 0}$ in $M(S)$ defined by

$$
T(t)=\left(\mathrm{e}^{t A_{n}}\right)^{\prime}
$$

that is $T(t)$ is the adjoint of $\mathrm{e}^{t A_{n}}$ in $M(S)$. This semigroup is in general not strongly continuous. However, if we let $X^{\odot}$ to be the subspace of $M(S)$ consisting of all $\mu$ such that $t \mapsto T(t) \mu$ is continuous at 0 , then the operators

$$
T(t)^{\odot}:=T(t)_{\mid X^{\odot}}, \quad t \geq 0
$$

form a strongly continuous semigroup in $X^{\odot}$, called the sun dual semigroup; see [9, Section II.2.6] for all details. The generator of this semigroup is the part $A_{n}^{\odot}$, see [9, p. 60], of the adjoint $A_{n}^{\prime}$ in $X^{\odot}$, that is

$$
A_{n}^{\odot} \mu=A_{n}^{\prime} \mu, \quad \mu \in D\left(A_{n}^{\odot}\right):=\left\{\mu \in D\left(A_{n}^{\prime}\right): A_{n}^{\prime} \mu \in X^{\odot}\right\} .
$$

The problem is that it is often difficult to characterize the space $X^{\odot}$. However, in our setup, the space $L^{1}(S)$ is much more natural to work with, because we can talk about dynamics of the densities of the underlying process. The space $L^{1}(S)$ is (isometrically isomorphic to) a closed subspace of $M(S)$, and for every $\varphi \in L^{1}(S)$ the measure $\mu_{\varphi}$ given by $\mu_{\varphi}(E):=\int_{E} \varphi \mathrm{d} m$ for all Borel measurable $E \subset S$, may be identified with $\varphi$. With slight abuse of notation we write $\mu_{\varphi} \in L^{1}(S)$ or even $\mu_{\varphi}=\varphi$. We prove, see Proposition 2.1 and Theorem 2.3, that $L^{1}(S)$ is a closed subspace of $X^{\odot}$ and that the part of $A_{n}^{\odot}$ in $L^{1}(S)$ is exactly $A_{n}^{*}$.

Proposition 2.1 Let $n \in \mathbb{N}$ and $A_{n}^{\prime}: M(S) \rightarrow M(S)$ be the adjoint operator of $A_{n}$ defined in $C(S)$ by (1.3). For every $\varphi \in L^{1}(S)$ we have $A_{n}^{\prime} \mu_{\varphi} \in L^{1}(S)$ and

$$
A_{n}^{\prime} \mu_{\varphi}=A_{n}^{*} \varphi
$$

where $A_{n}^{*}$ is given by (2.4).

To prove Proposition 2.1 we use the following lemma.

Lemma 2.2 Let $n \in \mathbb{N}$. If $f \in D\left(A_{n}\right)$ and $\varphi \in D\left(A_{n}^{*}\right)$, then 


$$
\int_{S}\left(A_{n} f\right) \varphi \mathrm{d} m=\int_{S}\left(A_{n}^{*} \varphi\right) f \mathrm{~d} m
$$

Proof Integrating by parts, for each $j \in \mathcal{N}$ the integral $\int_{E_{j}} \varphi f^{\prime \prime} \mathrm{d} m$ equals

$$
\varphi\left(R_{j}\right) f^{\prime}\left(R_{j}\right)-\varphi^{\prime}\left(R_{j}\right) f\left(R_{j}\right)+\varphi^{\prime}\left(L_{j}\right) f\left(L_{j}\right)-\varphi\left(L_{j}\right) f^{\prime}\left(L_{j}\right)+\int_{E_{j}} \varphi^{\prime \prime} f \mathrm{~d} m .
$$

Hence, equality (2.5) holds if and only if

$$
\sum_{j \in \mathcal{N}} \sigma_{j}\left[\varphi\left(R_{j}\right) f^{\prime}\left(R_{j}\right)-\varphi^{\prime}\left(R_{j}\right) f\left(R_{j}\right)+\varphi^{\prime}\left(L_{j}\right) f\left(L_{j}\right)-\varphi\left(L_{j}\right) f^{\prime}\left(L_{j}\right)\right]=0 .
$$

Since $f$ belongs to $D\left(A_{n}\right)$, transmission conditions (1.1) and (1.2) are satisfied. Thus (2.6) holds if and only if

$$
\begin{aligned}
& \kappa_{n}^{-1} \sum_{j \in \mathcal{N}} \sigma_{j} \varphi\left(R_{j}\right)\left[\sum_{i \neq j} r_{j i} f\left(R_{j i}\right)-r_{j} f\left(R_{j}\right)\right]-\sum_{j \in \mathcal{N}} \sigma_{j} \varphi^{\prime}\left(R_{j}\right) f\left(R_{j}\right) \\
& \quad+\kappa_{n}^{-1} \sum_{j \in \mathcal{N}} \sigma_{j} \varphi\left(L_{j}\right)\left[\sum_{i \neq j} l_{j i} f\left(L_{j i}\right)-l_{j} f\left(L_{j}\right)\right]+\sum_{j \in \mathcal{N}} \sigma_{j} \varphi^{\prime}\left(L_{j}\right) f\left(L_{j}\right)=0 .
\end{aligned}
$$

Changing the order of summation in the double sums, and changing the variable $j \in \mathcal{N}$ to $i \in \mathcal{N}$ in the single sums, the last equality becomes

$$
\begin{aligned}
\kappa_{n}^{-1} & \sum_{i \in \mathcal{N}} \sum_{j \neq i} \sigma_{j} r_{j i} \varphi\left(R_{j}\right) f\left(R_{j i}\right)-\sum_{i \in \mathcal{N}} \sigma_{i} f\left(R_{i}\right)\left[\kappa_{n}^{-1} r_{i} \varphi\left(R_{i}\right)+\varphi^{\prime}\left(R_{i}\right)\right] \\
& +\kappa_{n}^{-1} \sum_{i \in \mathcal{N}} \sum_{j \neq i} \sigma_{j} l_{j i} \varphi\left(L_{j}\right) f\left(L_{j i}\right)-\sum_{i \in \mathcal{N}} \sigma_{i} f\left(L_{i}\right)\left[\kappa_{n}^{-1} l_{i} \varphi\left(L_{i}\right)-\varphi^{\prime}\left(L_{i}\right)\right]=0 .
\end{aligned}
$$

Notice that $L_{j i}$ is either $L_{i}$ or $R_{i}$, or is left undefined, and the same holds for $R_{j i}$. Thus we can rewrite the last condition in the form

$$
\sum_{i \in \mathcal{N}} \sigma_{i} f\left(R_{i}\right)\left[\kappa_{n}^{-1} F_{R, i} \varphi-\varphi^{\prime}\left(R_{i}\right)\right]+\sum_{i \in \mathcal{N}} \sigma_{i} f\left(L_{i}\right)\left[\varphi^{\prime}\left(L_{i}\right)-\kappa_{n}^{-1} F_{L, i} \varphi\right]=0,
$$

which is true, since $\varphi$ satisfies the transition conditions (2.3).

Proof of Proposition 2.1 Let $\varphi \in L^{1}(S)$. Then

$$
\left(A_{n}^{\prime} \mu_{\varphi}\right) f=\int_{S} A_{n} f \mathrm{~d} \mu_{\varphi}=\int_{S}\left(A_{n} f\right) \varphi \mathrm{d} m,
$$

and Lemma 2.2 completes the proof. 


\subsection{Generation theorem in $L^{1}(S)$}

We know from [4, Proposition 2.1] that for each $n \in \mathbb{N}$ the operator $A_{n}$ generates a Feller semigroup in $C(S)$. We prove that the operator $A_{n}^{*}$ defined in Sect. 2.1 generates a sub-Markov semigroup $\left\{\mathrm{e}^{t A_{n}^{*}}\right\}_{t \geqslant 0}$ in $L^{1}(S)$. Moreover, if $\left\{\mathrm{e}^{t A_{n}}\right\}_{t \geqslant 0}$ is conservative, we show that $\left\{\mathrm{e}^{t A_{n}^{*}}\right\}_{t \geqslant 0}$ is Markov. The main theorem of this section is as follows.

Theorem 2.3 For each $n \in \mathbb{N}$ the operator $A_{n}^{*}$ generates a sub-Markov semigroup in $L^{1}(S)$. Moreover, if the semigroup generated by $A_{n}$ is conservative, then $A_{n}^{*}$ generates a Markov semigroup.

First we recall some basic information about one-dimensional Laplacian with homogeneous Neumann boundary conditions. Let $a, b \in \mathbb{R}$ be such that $a<b$, and let $G$ be the operator in $L^{1}(a, b)$ given by

$$
G \varphi:=\varphi^{\prime \prime}
$$

with the domain

$$
D(G):=\left\{\varphi \in W^{2,1}(a, b): \varphi^{\prime}(a)=\varphi^{\prime}(b)=0\right\} .
$$

Then, see for example [19, Proposition 2.1.2, Exercise 2.1.3.4] and [5, Corollary 32.1 , the following is true.

Proposition 2.4 The operator $G$ generates a strongly continuous semigroup of contractions in $L^{1}(a, b)$, and for every $\varphi \in L^{1}(a, b)$ we have

$$
\lim _{t \rightarrow+\infty} \mathrm{e}^{t G} \varphi=\frac{1}{b-a} \int_{a}^{b} \varphi(x) \mathrm{d} x
$$

in $L^{1}(a, b)$, where $\int_{a}^{b} \varphi(x) \mathrm{d} x$ is identified with the constant function on $(a, b)$.

To prove Theorem 2.3 we note first that $\lambda-A_{n}^{*}$ satisfies the range condition.

Lemma 2.5 For all $n \in \mathbb{N}$ and for sufficiently large $\lambda>0$ the image of $D\left(A_{n}^{*}\right)$ under the operator $\lambda-A_{n}^{*}$ equals $L^{1}(S)$.

We omit the proof of this lemma, because we establish the result in a much more general situation later on; see Step 2 of the proof of Theorem 2.6.

Proof of Theorem 2.3 The domain of $A_{n}^{*}$ is dense in $L^{1}(S)$, since for each $\varphi \in L^{1}(S)$ we may approximate $\varphi$ as restricted to the edge $E_{i}$ by a continuous function that is compactly supported in the interior of $E_{i}$. Moreover, for the adjoint operator $A_{n}^{\prime}$ of $A_{n}$ (see Sect. 2.1), and every $\lambda$ in the resolvent set $\rho\left(A_{n}\right)$, we have, see [25, Theorem 1.10.2], $\lambda \in \rho\left(A_{n}^{*}\right)$ and 


$$
\left[\left(\lambda-A_{n}\right)^{-1}\right]^{\prime}=\left(\lambda-A_{n}^{\prime}\right)^{-1}
$$

Since $A_{n}$ is the generator of a contraction semigroup by Theorem 1.1, we have $\left\|\left(\lambda-A_{n}\right)^{-1}\right\|_{\mathcal{L}(C(S))} \leqslant \lambda^{-1}$ for all $t \geq 0$ and $\lambda>0$. The fact that the norm of the adjoint equals the norm of the related operator, implies that

$$
\left\|\left(\lambda-A_{n}^{\prime}\right)^{-1}\right\|_{\mathcal{L}(M(S))} \leqslant \lambda^{-1}, \quad \lambda>0 .
$$

However, by Proposition 2.1,

$$
\left(\lambda-A_{n}^{\prime}\right) \varphi=\left(\lambda-A_{n}^{*}\right) \varphi, \quad \lambda>0, \varphi \in D\left(A_{n}^{*}\right),
$$

and consequently

$$
\left\|\left(\lambda-A_{n}^{*}\right) \varphi\right\|_{L^{1}(S)} \geqslant \lambda\|\varphi\|_{L^{1}(S)}, \quad \lambda>0, \varphi \in D\left(A_{n}^{*}\right) .
$$

Combining this with Lemma 2.5, we see that $\lambda-A_{n}^{*}$ is one-to-one and onto for some [hence all by (2.9)] $\lambda>0$ and

$$
\left\|\left(\lambda-A_{n}^{*}\right)^{-1}\right\|_{\mathcal{L}\left(L^{1}(S)\right)} \leq \lambda^{-1}
$$

By the Hille-Yosida theorem this shows that $A_{n}^{*}$ generates a strongly continuous semigroup of contractions in $L^{1}(S)$.

We are left with proving that the semigroup is sub-Markov. To this end we use the fact that, see for example [17, Corollary 7.8.1], the semigroup generated by an operator $A$ is sub-Markov, provided that the operator $\lambda(\lambda-A)^{-1}$ is sub-Markov for all $\lambda>0$. Hence, in our setting, we need to check that $\lambda\left(\lambda-A_{n}^{*}\right)^{-1}$ is sub-Markov for all $\lambda>0$. First we show that if $\varphi \in L^{1}(S)$ and $\varphi \geqslant 0$, then $\left(\lambda-A_{n}^{*}\right)^{-1} \varphi \geqslant 0$ for every $\lambda>0$. Recall that $m$ is the Lebesgue measure on $S$, and suppose, contrary to our claim, that there exists a function $\varphi \geqslant 0$, a set $\Gamma \subset S$ with $m(\Gamma)>0$, and a real number $\delta>0$ such that for some $\lambda_{0}>0$ we have $\left(\lambda_{0}-A_{n}^{*}\right)^{-1} \varphi \leqslant-\delta$ almost everywhere on $\Gamma$. Without loss of generality, we may assume that $\Gamma$ is a subset of some edge $E_{i}$. Then, for a given $\varepsilon>0$, we choose an open set $O \subset E_{i}$ and a closed set $\Gamma^{\prime}$ such that $\Gamma^{\prime} \subset \Gamma \subset O$ and $m\left(O \backslash \Gamma^{\prime}\right)<\varepsilon$. By the Urysohn lemma, there exists a continuous real function $0 \leqslant f \leqslant 1$ with $f \equiv 1$ on $\Gamma^{\prime}$ and $f \equiv 0$ outside $O$. Then

$$
\begin{aligned}
\int_{S} f\left(\lambda_{0}-A_{n}^{*}\right)^{-1} \varphi \mathrm{d} m & =\int_{\Gamma^{\prime}} f\left(\lambda_{0}-A_{n}^{*}\right)^{-1} \varphi \mathrm{d} m+\int_{O \backslash \Gamma^{\prime}} f\left(\lambda_{0}-A_{n}^{*}\right)^{-1} \varphi \mathrm{d} m \\
& \leqslant-\delta m\left(\Gamma^{\prime}\right)+\int_{O \backslash \Gamma^{\prime}} f\left(\lambda_{0}-A_{n}^{*}\right)^{-1} \varphi \mathrm{d} m .
\end{aligned}
$$

Since $\varepsilon$ is arbitrary small, it follows that the left-hand side is strictly negative. However, by (2.8),

$$
\int_{S} f\left(\lambda_{0}-A_{n}^{*}\right)^{-1} \varphi \mathrm{d} m=\int_{S} \varphi\left(\lambda_{0}-A_{n}\right)^{-1} f \mathrm{~d} m \geqslant 0,
$$


where the inequality is a consequence of the fact that $A_{n}$ generates a Feller semigroup. This leads to a contradiction and proves that $\left(\lambda-A_{n}^{*}\right)^{-1}$ is a positive operator for each $\lambda>0$.

Let now $\varphi \in L^{1}(S)$. Since $A_{n}$ generates a Feller semigroup, we have

$$
\left(\lambda-A_{n}\right)^{-1} \mathbb{1}_{S}=\int_{0}^{\infty} \mathrm{e}^{-\lambda t} \mathrm{e}^{t A_{n}} \mathbb{1}_{S} \mathrm{~d} t \leqslant \mathbb{1}_{S} \int_{0}^{\infty} \mathrm{e}^{-\lambda t} \mathrm{~d} t=\lambda^{-1} \mathbb{1}_{S}, \quad \lambda>0,
$$

where $\mathbb{1}_{S} \equiv 1$ on $S$. Thus, by (2.8),

$$
\int_{S} \lambda\left(\lambda-A_{n}^{*}\right)^{-1} \varphi \mathrm{d} m=\int_{S} \varphi \lambda\left(\lambda-A_{n}\right)^{-1} \mathbb{1}_{S} \mathrm{~d} m \leqslant \int_{S} \varphi \mathrm{d} m
$$

for all $\lambda>0$, which completes the proof of the sub-Markov property.

Finally, for the last part of the theorem, if we assume that the semigroup generated by $A_{n}$ is conservative, then inequality in (2.10) becomes equality, and $\int_{S} \lambda\left(\lambda-A_{n}^{*}\right)^{-1} \varphi \mathrm{d} m=\int_{S} \varphi \mathrm{d} m$ for all $\lambda>0$ and $\varphi \in L^{1}(S)$, showing that the semigroup generated by $A_{n}^{*}$ is Markov.

\subsection{Generalization of the generation theorem}

In this section we generalize the generation part of Theorem 2.3 to the case, where $F_{L, i}$ and $F_{R, i}$ from transmission conditions (2.3) are more general functionals. To this end we cannot, in contradistinction to the proof of Theorem 2.3, use the fact that a pre-dual operator generates a semigroup in $C(S)$, and we need to prove the result directly.

Let $\sigma$ be as in Sect. 1.1. We define the operator $A$ in $L^{1}(S)$ by the formula

$$
A \varphi:=\sigma \varphi^{\prime \prime}, \quad \varphi \in D(A):=W^{2,1}(S) .
$$

Now we introduce the operator $A_{F}$ in $L^{1}(S)$ as

$$
A_{F} \varphi:=A \varphi, \quad \varphi \in D\left(A_{F}\right),
$$

with the domain

$$
D\left(A_{F}\right):=\left\{\varphi \in W^{2,1}(S): L \varphi=F \varphi\right\}
$$

where

$$
L \varphi:=\left(\varphi^{\prime}\left(L_{i}\right), \varphi^{\prime}\left(R_{i}\right)\right)_{i \in \mathcal{N}}, \quad F \varphi:=\left(F_{L, i} \varphi, F_{R, i} \varphi\right)_{i \in \mathcal{N}}
$$

We consider $L$ and $F$ as linear operators $L^{1}(S) \rightarrow \mathbb{R}^{2 N}$ with domains

$$
D(L)=W^{2,1}(S), \quad D(F)=W^{1,1}(S)
$$

here $F_{L, i}, F_{R, i}$ are linear functionals acting on $W^{1,1}(S)$, however we do not assume that $F$ is bounded in $\left(W^{1,1}(S),\|\cdot\|_{W^{1,1}(S)}\right)$. We may write 


$$
A_{F}=A_{\mid \operatorname{ker}(L-F)},
$$

that is $A_{F}$ is the restriction of $A$ to the kernel of $L-F$. In particular, if we let $F=0$, then we consider $A_{0}$ as $A$ restricted to the kernel of $L$, that is

$$
A_{0}=A_{\mid \operatorname{ker} L} .
$$

It is well known, see [19, Proposition 2.1.2, Exercise 2.1.3.4], that the semigroup generated by the operator $G$ from Proposition 2.4 is bounded holomorphic of angle $\pi / 2$, which implies that so is the semigroup generated by $A_{0}$; this semigroup is isomorphic to the tensor product of semigroups generated by (rescaled) $G$ given by (2.7), see [21, p. 21]. Consequently, see [1, Corollary 3.7.12],

$$
\sup _{\operatorname{Re} \lambda>0}\left\|\lambda\left(\lambda-A_{0}\right)^{-1}\right\|_{\mathcal{L}\left(L^{1}(S)\right)}<+\infty .
$$

Finally, we introduce the operator

$$
L_{\lambda}: \mathbb{R}^{2 N} \rightarrow \operatorname{ker}(\lambda-A) \subset W^{2,1}(S)
$$

as the inverse of $L$ restricted to the kernel of $\lambda-A$, that is

$$
L_{\lambda}:=\left(L_{\mid \operatorname{ker}(\lambda-A)}\right)^{-1} \text {. }
$$

Such inverse exists for all $\lambda \in \mathbb{C} \backslash(-\infty, 0]$, which follows by [14, Lemma 1.2].

Now we are ready to state the main result.

Theorem 2.6 Assume that there exists $r>0$ such that

$$
\sup _{\operatorname{Re} \lambda>r}\left\|F\left(\lambda-A_{0}\right)^{-1}\right\|_{\mathcal{L}\left(L^{1}(S), \mathbb{R}^{2 N}\right)}<+\infty,
$$

and

$$
\sup _{\operatorname{Re} \lambda>r}\left\|F L_{\lambda}\right\|_{\mathcal{L}\left(\mathbb{R}^{2 N}\right)}<1 .
$$

Then the operator $A_{F}$ defined by (2.11) generates a holomorphic semigroup in $L^{1}(S)$.

As a simple consequence of Theorem 2.6 we obtain the following corollary, improving [3, Theorem 2.4], where one needs to assume that, using our notation, $F_{L, i} \varphi$ and $F_{R, i} \varphi$ depend only on the values of $\varphi$ at the vertices.

For $\kappa \geq 1$ let

$$
A_{\kappa, F}:=\kappa A_{\kappa^{-1} F},
$$

where $A_{\kappa^{-1} F}$ is given by (2.11) with $F$ replaced with $\kappa^{-1} F$.

Corollary 2.7 Assume that the operator $F$ given by (2.12) is bounded as $W^{1,1}(S) \rightarrow \mathbb{R}^{2 N}$, that is 


$$
\|F \varphi\|_{\mathbb{R}^{2 N}} \leq C_{F}\|\varphi\|_{W^{1,1}(S)}, \quad \varphi \in W^{1,1}(S)
$$

for some $C_{F}>0$. Then the operator $A_{\kappa, F}$ generates a holomorphic semigroup in $L^{1}(S)$, and there exists $\omega \geq 0$, that does not depend on $\kappa$, such that

$$
\sup _{t \geq 0, \kappa \geq 1}\left\|\mathrm{e}^{-\omega t} \mathrm{e}^{t A_{\kappa, F}}\right\|_{\mathcal{L}\left(L^{1}(S)\right)}<+\infty .
$$

Before we prove Theorem 2.6 and Corollary 2.7, we establish some properties of the operator $L_{\lambda}$. For simplicity of notation we introduce for every $\mu \in \mathbb{C}$ the function $\mathrm{e}_{\mu}$ by the formula $\mathrm{e}_{\mu}(x)=\mathrm{e}^{\mu x}$.

Lemma 2.8 Let $a, d>0$ and $\alpha, \beta \in \mathbb{R}$. For every $\lambda \in \mathbb{C} \backslash(-\infty, 0]$ there exists a unique $\varphi \in W^{2,1}(0, d)$ satisfying

$$
\left\{\begin{array}{l}
\lambda \varphi-a \varphi^{\prime \prime}=0, \\
\varphi^{\prime}(0)=\alpha, \\
\varphi^{\prime}(d)=\beta,
\end{array}\right.
$$

which is given by

$$
\varphi=c_{1} \mathrm{e}_{\mu}+c_{2} \mathrm{e}_{-\mu},
$$

where $\mu$ is the square root of $\lambda / a$ with positive real part, and

$$
c_{1}:=\frac{1}{\mu} \frac{\beta-\alpha \mathrm{e}^{-\mu d}}{\mathrm{e}^{\mu d}-\mathrm{e}^{-\mu d}}, \quad c_{2}:=\frac{1}{\mu} \frac{\beta-\alpha \mathrm{e}^{\mu d}}{\mathrm{e}^{\mu d}-\mathrm{e}^{-\mu d}},
$$

Moreover, for every $r>0$ there exists $C>0$ that depend merely on $a, d$ and $r$, such that for all $\lambda$ satisfying $\operatorname{Re} \lambda>r$, we have

$$
\|\varphi\|_{L^{1}(0, d)} \leq \frac{C}{|\lambda|}\|(\alpha, \beta)\|_{\mathbb{R}^{2}} .
$$

Proof We obtain the explicit formula for $\varphi$ using methods from classical ODE theory. For the estimate (2.20), we note that $\left|\mathrm{e}^{\mu d}-\mathrm{e}^{-\mu d}\right| \geq \mathrm{e}^{d \operatorname{Re} \mu}-\mathrm{e}^{-d \operatorname{Re} \mu}$, and

$$
\int_{0}^{d}\left|\mathrm{e}^{\mu x}\right| \mathrm{d} x=\int_{0}^{d} \mathrm{e}^{x \operatorname{Re} \mu} \mathrm{d} x=\frac{1}{\operatorname{Re} \mu}\left(\mathrm{e}^{d \operatorname{Re} \mu}-1\right) .
$$

Therefore, using the fact that $|\mu| \leq \operatorname{Re} \mu / \sqrt{2}$, provided that $\operatorname{Re} \lambda>r>0$, the claim follows.

Corollary 2.9 For all $\lambda \in \mathbb{C} \backslash(-\infty, 0]$ the operator $L_{\lambda}$ given by (2.15) satisfies

$$
A L_{\lambda}=\lambda L_{\lambda}, \quad \lambda \in \mathbb{C} \backslash(-\infty, 0] .
$$

Moreover, for all $r>0$ there exists $C_{L}>0$ such that for all $\lambda$ satisfying $\operatorname{Re} \lambda>r$, we have 


$$
\left\|L_{\lambda}\right\|_{\mathcal{L}\left(\mathbb{R}^{2 N}, L^{1}(S)\right)} \leq \frac{C_{L}}{|\lambda|}
$$

and

$$
\left\|L_{\lambda}\right\|_{\mathcal{L}\left(\mathbb{R}^{2 N}, W^{1,1}(S)\right)} \leq \frac{C_{L}}{\sqrt{|\lambda|}} .
$$

Proof Given $\left(\alpha_{i}, \beta_{i}\right)_{i \in \mathcal{N}} \in \mathbb{R}^{2 N}$, when solving

$$
L \varphi=\left(\alpha_{i}, \beta_{i}\right)_{i \in \mathcal{N}}
$$

for $\varphi \in \operatorname{ker}(\lambda-A)$, for each $i \in \mathcal{N}$ we apply Lemma 2.8. For the estimate of $L_{\lambda}$ as $\mathbb{R}^{2 N} \rightarrow W^{1,1}(S)$ we differentiate (2.19) and use (2.20).

With such a preparation we are ready to prove Theorem 2.6. To this end we use Greiner-like perturbation approach. Note that the method used in the proof of [14, Lemma 1.4] does not work here, however, see Remark 2.10).

Proof of Theorem 2.6 We fix $r>0$ such that (2.16) and (2.17) hold. By [1, Proposition 3.7.4, Corollary 3.7.12] it suffices to show that there exists $r^{\prime}>0$ such that every $\lambda$ satisfying $\operatorname{Re} \lambda>r^{\prime}$ belongs to the resolvent set of $A_{F}$, and that

$$
\sup _{\operatorname{Re} \lambda>r^{\prime}}\left\|\lambda\left(\lambda-A_{F}\right)^{-1}\right\|_{\mathcal{L}\left(L^{1}(S)\right)}<+\infty .
$$

Step 1 We prove that the operator $\lambda-A_{F}$ is one-to-one for every $\lambda$ satisfying $\operatorname{Re} \lambda>r$. Fix such $\lambda$, and let $\mathcal{A}, \mathcal{F}$ be the operators in the space $L^{1}(S) \times \mathbb{R}^{2 N}$ given by

$$
\mathcal{A}(\varphi, 0):=(A \varphi,-L \varphi), \quad \mathcal{F}(\varphi, 0):=(0, F \varphi)
$$

for all $\varphi \in D(A)$, with domains

$$
D(\mathcal{A})=D(\mathcal{F}):=D(A) \times\{0\} .
$$

The operator $\lambda-\mathcal{A}$ is one-to-one and onto, and

$$
(\lambda-\mathcal{A})^{-1}(\varphi, \alpha)=\left(\left(\lambda-A_{0}\right)^{-1} \varphi+L_{\lambda} \alpha, 0\right), \quad(\varphi, \alpha) \in L^{1}(S) \times \mathbb{R}^{2 N} .
$$

Therefore, denoting by $\mathcal{I}$ the identity operator in $L^{1}(S) \times \mathbb{R}^{2 N}$, for all $(\varphi, \alpha) \in L^{1}(S) \times \mathbb{R}^{2 N}$ we have

$$
\mathcal{I}(\varphi, \alpha)-\mathcal{F}(\lambda-\mathcal{A})^{-1}(\varphi, \alpha)=\left(\varphi,-F\left(\lambda-A_{0}\right)^{-1} \varphi+\left(I_{\mathbb{R}^{2 N}}-F L_{\lambda}\right) \alpha\right) .
$$

Because $I_{\mathbb{R}^{2 N}}-F L_{\lambda}$ is invertible, which is a consequence of (2.17), the operator $\mathcal{I}-\mathcal{F}(\lambda-\mathcal{A})^{-1}$ is one-to-one and onto, with the inverse

$$
\left(\mathcal{I}-\mathcal{F}(\lambda-\mathcal{A})^{-1}\right)^{-1}(\varphi, \alpha)=\left(\varphi,\left(I_{\mathbb{R}^{2 N}}-F L_{\lambda}\right)^{-1}\left(F\left(\lambda-A_{0}\right)^{-1} \varphi+\alpha\right)\right)
$$


for all $(\varphi, \alpha) \in L^{1}(S) \times \mathbb{R}^{2 N}$. Since $\lambda-\mathcal{A}-\mathcal{F}=\left(\mathcal{I}-\mathcal{F}(\lambda-\mathcal{A})^{-1}\right)(\lambda-\mathcal{A})$, it follows that the operator $\lambda-\mathcal{A}-\mathcal{F}$ is one-to-one and onto, and

$$
(\lambda-\mathcal{A}-\mathcal{F})^{-1}=(\lambda-\mathcal{A})^{-1}\left(\mathcal{I}-\mathcal{F}(\lambda-\mathcal{A})^{-1}\right)^{-1} .
$$

However, for every $\varphi \in D\left(A_{F}\right)$ we have

$$
(\lambda-\mathcal{A}-\mathcal{F})(\varphi, 0)=((\lambda-A) \varphi, L \varphi-F \varphi)=\left(\left(\lambda-A_{F}\right) \varphi, 0\right),
$$

which shows that the operator $\lambda-A_{F}$ is one-to-one, and

$$
\varphi=\left[\left(I_{L^{1}(S)}+L_{\lambda}\left(I_{\mathbb{R}^{2 N}}-F L_{\lambda}\right)^{-1} F\right)\left(\lambda-A_{0}\right)^{-1}\right]\left(\lambda-A_{F}\right) \varphi .
$$

Step 2 For $\lambda$ as in Step 1 we prove that the operator $\lambda-A_{F}$ is onto. Given $\psi \in L^{1}(S)$ let, see (2.23),

$$
\varphi:=\left(I_{L^{1}(S)}+L_{\lambda}\left(I_{\mathbb{R}^{2 N}}-F L_{\lambda}\right)^{-1} F\right)\left(\lambda-A_{0}\right)^{-1} \psi .
$$

We have $\left(\lambda-A_{0}\right)^{-1} \psi \in W^{2,1}(S)$, and since $L_{\lambda}$ transforms $\mathbb{R}^{2 N}$ into $W^{2,1}(S)$, $\varphi$ belongs to $W^{2,1}(S)$. Moreover, since $\left(\lambda-A_{0}\right)^{-1} \psi \in D\left(A_{0}\right)$, it follows that $L \varphi=\left(I_{\mathbb{R}^{2 N}}-F L_{\lambda}\right)^{-1} F\left(\lambda-A_{0}\right)^{-1} \psi$. Consequently

$$
\begin{aligned}
L \varphi & =F\left(\left(\lambda-A_{0}\right)^{-1} \psi+L_{\lambda} L \varphi\right) \\
& =F\left(\left(\lambda-A_{0}\right)^{-1} \psi+L_{\lambda}\left(I_{\mathbb{R}^{2 N}}-F L_{\lambda}\right)^{-1} F\left(\lambda-A_{0}\right)^{-1} \psi\right) \\
& =F \varphi .
\end{aligned}
$$

This proves that $\varphi \in D\left(A_{F}\right)$. Moreover, because $A_{F} \eta=A \eta$ for all $\eta \in D\left(A_{F}\right)$, by (2.21) it follows that

$$
\begin{aligned}
A_{F} \varphi & =A_{0}\left(\lambda-A_{0}\right)^{-1} \psi+A L_{\lambda}\left(I_{\mathbb{R}^{2 N}}-F L_{\lambda}\right)^{-1} F\left(\lambda-A_{0}\right)^{-1} \psi \\
& =\lambda\left(\lambda-A_{0}\right)^{-1} \psi-\psi+\lambda L_{\lambda}\left(I_{\mathbb{R}^{2 N}}-F L_{\lambda}\right)^{-1} F\left(\lambda-A_{0}\right)^{-1} \psi \\
& =\lambda \varphi-\psi,
\end{aligned}
$$

or equivalently $\left(\lambda-A_{F}\right) \varphi=\psi$.

Step 3 By Step 1 and Step 2 we know that

$$
\left(\lambda-A_{F}\right)^{-1}=\left[I_{L^{1}(S)}+L_{\lambda}\left(I_{\mathbb{R}^{2 N}}-F L_{\lambda}\right)^{-1} F\right]\left(\lambda-A_{0}\right)^{-1}
$$

for all $\operatorname{Re} \lambda>r$. We have

$$
\begin{aligned}
& \left\|L_{\lambda}\left(I_{\mathbb{R}^{2 N}}-F L_{\lambda}\right)^{-1} F\left(\lambda-A_{0}\right)^{-1}\right\|_{\mathcal{L}\left(L^{1}(S)\right)} \\
& \quad \leq\left\|L_{\lambda}\right\|_{\mathcal{L}\left(\mathbb{R}^{2 N}, L^{1}(S)\right)}\left\|\left(I_{\mathbb{R}^{2 N}}-F L_{\lambda}\right)^{-1}\right\|_{\mathcal{L}\left(\mathbb{R}^{2 N}\right)}\left\|F\left(\lambda-A_{0}\right)^{-1}\right\|_{\mathcal{L}\left(L^{1}(S), \mathbb{R}^{2 N}\right)},
\end{aligned}
$$

hence, by (2.16), (2.17) and Corollary 2.9, it follows that

$$
\sup _{\operatorname{Re} \lambda>r}\left\|\lambda L_{\lambda}\left(I_{\mathbb{R}^{2 N}}-F L_{\lambda}\right)^{-1} F\left(\lambda-A_{0}\right)^{-1}\right\|_{\mathcal{L}\left(L^{1}(S)\right)}<+\infty .
$$

Using (2.14) and (2.24), this proves (2.22). 
Finally, we use Theorem 2.6 to prove Corollary 2.7.

Proof of Corollary 2.7 For the generation part, by Theorem 2.6, we are left with proving that (2.16)-(2.17) hold with $F, A_{0}$ and $L_{\lambda}$ replaced by $\kappa^{-1} F, \kappa A_{0}$ and $L_{\lambda, \kappa}$, respectively; here $L_{\lambda, \kappa}:=\left(L_{\mid \operatorname{ker}(\lambda-\kappa A)}\right)^{-1}=L_{\kappa^{-1} \lambda}$.

Fix $r>0$ and $\kappa \geq 1$. A straightforward computation, similar to those in the proof of Lemma 2.8, shows that there exists $C>0$ such that

$$
\left\|\left(\lambda-A_{0}\right)^{-1} \varphi\right\|_{W^{1,1}(S)} \leq \frac{C}{\sqrt{|\lambda|}}\|\varphi\|_{L^{1}(S)}, \quad \operatorname{Re} \lambda>r, \varphi \in L^{1}(S) .
$$

Hence, by (2.18) and the fact that $\left(\lambda-\kappa A_{0}\right)^{-1}=\kappa^{-1}\left(\kappa^{-1} \lambda-A_{0}\right)^{-1}$, we obtain

$$
\left\|\kappa^{-1} F\left(\lambda-\kappa A_{0}\right)^{-1}\right\|_{\mathcal{L}\left(L^{1}(S), \mathbb{R}^{2 N}\right)} \leq \frac{C_{F} C}{\sqrt{\kappa^{3}|\lambda|}}
$$

for all $\operatorname{Re} \lambda>r$. Similarly, by Corollary 2.9,

$$
\left\|\kappa^{-1} F L_{\kappa^{-1} \lambda}\right\|_{\mathcal{L}\left(\mathbb{R}^{2 N}\right)} \leq \frac{C_{F} C_{L}}{\sqrt{\kappa|\lambda|}}, \quad \operatorname{Re} \lambda>r .
$$

Choosing $r^{\prime}>r$ such that the right-hand side is less than 1, it follows by Theorem 2.6 that $A_{\kappa, F}$ generates a holomorphic semigroup in $L^{1}(S)$.

In order to obtain a growth bound for $\mathrm{e}^{t A_{\kappa, F}}$, fix $r^{\prime}>0$ as above, and note that by (2.24) we have

$$
\left(\lambda-A_{\kappa, F}\right)^{-1}=\left[I_{L^{1}(S)}+L_{\kappa^{-1} \lambda}\left(I_{\mathbb{R}^{2 N}}-\kappa^{-1} F L_{\kappa^{-1} \lambda}\right)^{-1} \kappa^{-1} F\right]\left(\lambda-\kappa A_{0}\right)^{-1}
$$

for all $\lambda$ satisfying $\operatorname{Re} \lambda>r^{\prime}$. By (2.26) there exists $C^{\prime}>0$, that does not depend on $\kappa$ (recall that $\kappa \geq 1$ ), such that

$$
\left\|\left(I_{\mathbb{R}^{2 N}}-\kappa^{-1} F L_{\kappa^{-1} \lambda}\right)^{-1}\right\|_{\mathcal{L}\left(\mathbb{R}^{2 N}\right)} \leq C^{\prime}, \quad \operatorname{Re} \lambda>r^{\prime} .
$$

Therefore, by (2.14), (2.25) and Corollary 2.9 it follows that there exists $C^{\prime \prime}>0$, that does not depend on $\kappa$, such that

$$
\left\|\left(\lambda-A_{\kappa, F}\right)^{-1}\right\|_{\mathcal{L}\left(L^{1}(S)\right)} \leq \frac{C^{\prime \prime}}{|\lambda|}, \quad \operatorname{Re} \lambda>r^{\prime}
$$

This shows, see Theorems 2.6.1, 3.7.11 and the proof of Corollary 3.7.12 in [1], that there exist $\omega \geq 0$ and $M \geq 0$, both independent of $\kappa$, such that

$$
\left\|\mathrm{e}^{-\omega t} \mathrm{e}^{t A_{\kappa, F}}\right\|_{\mathcal{L}\left(L^{1}(S)\right)} \leq M, \quad t \geq 0
$$

which proves the claim.

Remark 2.10 We stress that it is not possible to prove Theorem 2.6 or Corollary 2.7 using Greiner's [14, Lemma 1.4], and we cannot hope for the relation 


$$
\left(\lambda-A_{F}\right)^{-1}=\left(I_{L^{1}(S)}-L_{\lambda} F\right)^{-1}\left(\lambda-A_{0}\right)^{-1}
$$

to hold. The reason is that $F$ is not a bounded operator in $L^{1}(S)$, and consequently $I_{L^{1}(S)}-L_{\lambda} F$ is not even defined on $L^{1}(S)$. However,

$$
\left(I_{L^{1}(S)}-L_{\lambda} F\right)^{-1}=\left[I_{L^{1}(S)}+L_{\lambda}\left(I_{\mathbb{R}^{2 N}}-F L_{\lambda}\right)^{-1} F\right]^{-1},
$$

provided that both sides are well defined. Assuming (2.17) or (2.18), we prove that the right-hand side exists. The proof relies on Nickel's approach [22] to boundary perturbations, however the idea that we can use it in an unbounded case is described in [15, Theorem 1] or [26, Lemma 3.4, p. 81].

\subsection{Convergence in $L^{1}(S)$}

In the setting as in Sect. 2.3, for every $n \in \mathbb{N}$ let $A_{n}$ be the operator defined in $L^{1}(S)$ by

$$
A_{n} \varphi:=A_{\kappa_{n}, F} \varphi=\kappa_{n} \sigma \varphi^{\prime \prime}, \quad \varphi \in D\left(A_{n}\right),
$$

where

$$
D\left(A_{n}\right):=\left\{\varphi \in W^{2,1}(S): \kappa_{n} L \varphi=F \varphi\right\}
$$

here $L$ and $F=\left(F_{L, i}, F_{R, i}\right)_{i \in \mathcal{N}}$ are defined by (2.12). We additionally assume that

$$
F_{L, i} \varphi=\int_{S} \varphi \mathrm{d} v_{L, i}, \quad F_{R, i} \varphi=\int_{S} \varphi \mathrm{d} v_{R, i}, \quad \varphi \in W^{1,1}(S),
$$

for some finite signed regular Borel measures $v_{L, i}$ and $v_{R, i}$ on $S$. The functionals are bounded as $W^{1,1}(S) \rightarrow \mathbb{R}$, see the proof of Lemma 2.13 , hence by Corollary 2.7 each $A_{n}$ generates a strongly continuous semigroup in $L^{1}(S)$ and there exists $\omega \geq 0$ and $M \geq 0$ such that

$$
\left\|\mathrm{e}^{-\omega t} \mathrm{e}^{t A_{n}}\right\|_{\mathcal{L}\left(L^{1}(S)\right)} \leq M, \quad n \in \mathbb{N}, t \geq 0 .
$$

The main result of this section is as follows.

Let $P$ be the projection in $L^{1}(S)$ given by

$$
P \varphi:=\left(d_{i}^{-1} \int_{E_{i}} \varphi \mathrm{d} m\right)_{i \in \mathcal{N}^{\prime}}, \quad \varphi \in L^{1}(S) .
$$

The range of $P$ is the closed subspace $L_{0}^{1}(S)$ of $L^{1}(S)$ consisting of all functions that are constant on each edge. We note that $L_{0}^{1}(S)$ is isometrically isomorphic to $\left(\mathbb{R}^{N},\|\cdot\|_{\mathbb{R}^{N}}\right)$ via the map

$$
\mathcal{I}: \varphi \mapsto\left(d_{i} \varphi_{i}\right)_{i \in \mathcal{N}}, \quad \varphi \in L_{0}^{1}(S),
$$


where $\varphi_{i}$ is the value of $\varphi \in L_{0}^{1}(S)$ on the edge $E_{i}$. Moreover, let $Q$ be the bounded operator in $L_{0}^{1}(S)$ given by

$$
Q \varphi=\left(d_{i}^{-1}\left(F_{R, i} \varphi-F_{L, i} \varphi\right)\right)_{i \in \mathcal{N}^{\prime}}, \quad \varphi \in L_{0}^{1}(S) .
$$

Theorem 2.11 For the generators $A_{n}, n \in \mathbb{N}$, given by (2.27), we have

$$
\lim _{n \rightarrow+\infty} \mathrm{e}^{t A_{n}} \varphi=\mathrm{e}^{t Q} P \varphi, \quad \varphi \in L^{1}(S), t>0
$$

in $L^{1}(S)$. The convergence is uniform on compact subsets of $(0, \infty)$. If $\varphi \in L_{0}^{1}(S)$, then (2.33) holds also for $t=0$, and the convergence is uniform on compact subsets of $[0,+\infty)$.

\subsection{Kurtz's Theorem}

To prove Theorem 2.11 we begin with a theorem due to Kurtz (see [10, Theorem 1.7.6] for a general version, or [5, Theorem 42.2] for the one used in the paper).

For each $n \in \mathbb{N}$ let $\mathcal{A}_{n}$ be the generator of a strongly continuous semigroup $\left\{\mathrm{e}^{t \mathcal{A}_{n}}\right\}_{t \geqslant 0}$ in a Banach space $\mathbb{X}$. Assume that the semigroups are equibounded, that is

$$
\left\|\mathrm{e}^{t \mathcal{A}_{n}}\right\|_{\mathcal{L}(\mathbb{X})} \leqslant C, \quad n \in \mathbb{N}, t \geqslant 0
$$

for some $C>0$. Denote by $\mathcal{A}_{\text {ex }}$ the extended limit of $\left(\mathcal{A}_{n}\right)_{n \in \mathbb{N}}$, that is the multivalued operator in $\mathbb{X}$ with the domain $D\left(\mathcal{A}_{\text {ex }}\right)$ composed of all $x \in \mathbb{X}$ such that there exists a sequence $\left(x_{n}\right)_{n \in \mathbb{N}}$ in $\mathbb{X}$ that converges to $x$ while the limit of $\mathcal{A}_{n} x_{n}$ exists as $n \rightarrow+\infty$. By $(x, y) \in \mathcal{A}_{\mathrm{ex}}$ we mean that $x \in D\left(\mathcal{A}_{\mathrm{ex}}\right)$ and $\lim _{n \rightarrow+\infty} \mathcal{A}_{n} x_{n}=y$ for some sequence $\left(x_{n}\right)_{n \in \mathbb{N}}$ in $D\left(\mathcal{A}_{\text {ex }}\right)$ converging to $x$. Moreover, assume that $\left(\varepsilon_{n}\right)_{n \in \mathbb{N}}$ is a sequence of positive real numbers converging to 0 , and denote by $\mathcal{A}_{\text {ex }}^{0}$ the extended limit of $\left(\varepsilon_{n} \mathcal{A}_{n}\right)_{n \in \mathbb{N}}$.

Suppose also that an operator $\mathcal{A}_{0}$ with domain $D\left(\mathcal{A}_{0}\right)$ generates a strongly continuous semigroup $\left\{\mathrm{e}^{t \mathcal{A}_{0}}\right\}_{t \geqslant 0}$ in $\mathbb{X}$ such that $\left\|\mathrm{e}^{t \mathcal{A}_{0}}\right\|_{\mathcal{L}(\mathbb{X})} \leqslant C$, and that for every $x \in \mathbb{X}$ the limit

$$
\lim _{t \rightarrow \infty} \mathrm{e}^{t \mathcal{A}_{0}} x=: \mathcal{P} x
$$

exists. The operator $\mathcal{P}$ is bounded, and $\mathcal{P}^{2}=\mathcal{P}$, since $\left\{\mathrm{e}^{t \mathcal{A}_{0}}\right\}_{t \geq 0}$ is a strongly continuous semigroup. Hence the range

$$
\mathbb{Y}:=\operatorname{range} \mathcal{P},
$$

is a closed subspace of $\mathbb{X}$. With this setup we use a special case of Kurtz's theorem.

Theorem 2.12 [5, Theorem 42.2] Let $\mathcal{A}$ be an operator in $\mathbb{X}$ such that $\mathbb{Y}$ is a subset of its domain. Assume that 
(i) if $x \in \mathbb{Y}$, then $(x, \mathcal{A} x) \in \mathcal{A}_{\mathrm{ex}}$,

(ii) if $y \in D\left(\mathcal{A}_{0}\right)$, then $\left(y, \mathcal{A}_{0} y\right) \in \mathcal{A}_{\mathrm{ex}}^{0}$,

(iii) the operator $\mathcal{P} \mathcal{A}$ with domain $\mathbb{Y}$ generates a strongly continuous semigroup in $\bigvee$.

Then for every $x \in \mathbb{X}$ and $t>0$,

$$
\lim _{n \rightarrow+\infty} \mathrm{e}^{t \mathcal{A}_{n}} x=\mathrm{e}^{t \mathcal{P A}} \mathcal{P} x
$$

in $\mathbb{X}$, and the convergence is uniform on compact subsets of $(0, \infty)$. If $x \in \mathbb{Y}$, then the formula holds also for $t=0$, and the convergence is uniform on compact subsets of $[0,+\infty)$.

\subsection{Proof of Theorem 2.11}

First we prove that the domains of the operators $A_{n}$ and $A_{0}$ are isomorphic, see Lemma 2.14. To this end for each $i \in \mathcal{N}$ let us choose $h_{L, i}, h_{R, i} \in W^{2,1}\left(E_{i}\right)$ satisfying

$$
F_{L, i} h_{L, i}=F_{R, i} h_{R, i}=0
$$

and

$$
h_{L, i}^{\prime}\left(L_{i}\right)=h_{R, i}^{\prime}\left(R_{i}\right)=1, \quad h_{L, i}^{\prime}\left(R_{i}\right)=h_{R, i}^{\prime}\left(L_{i}\right)=0
$$

The existence of such functions is a consequence of the following result.

Lemma 2.13 Let $d>0$, and let $v$ be a finite signed regular Borel measure on $[0, d]$. Then

$$
F_{\nu} \varphi:=\int_{0}^{d} \varphi \mathrm{d} \nu, \quad \varphi \in W^{1,1}(0, d)
$$

is a bounded linear functional on $W^{1,1}(0, d)$, and there exist $g, h \in W^{2,1}(0, d)$ such that

$$
F_{\nu} g=0, \quad g^{\prime}(0)=1, \quad g^{\prime}(d)=0
$$

and

$$
F_{\nu} h=0, \quad h^{\prime}(0)=0, \quad h^{\prime}(d)=1 .
$$

Proof We have

$$
\left|F_{\nu} \varphi\right| \leq\|\varphi\|_{L^{\infty}(0, d)}|\nu|([0, d]) \leq\|\varphi\|_{W^{1,1}(0, d)}|\nu|([0, d]), \quad \varphi \in W^{1,1}(0, d),
$$

where $\|\cdot\|_{L^{\infty}(0, d)}$ is the essential supremum norm, and $|v|$ is the total variation of $v$. This implies that $F_{v}$ is bounded. 
For the second part of the lemma, the existence of $g$ is clear, provided that $v$ is of the form $c \delta_{0}$ for some $c \in \mathbb{R}$, where $\delta_{0}$ is the Dirac measure at 0 . Therefore, assume that $v$ is not of that form. There exists $a \in(0, d)$ such that $v([a, d]) \neq 0$. Indeed, if $v([a, d])=0$ for all $a \in(0, d)$, then $v([a, b))$ for all $0<a<b \leq d$. This proves that $\nu(B)=0$ for all Borel sets $B \subset(0, d)$, hence $v$ is concentrated on $\{0, d\}$. However, by the continuity of measure, we have

$$
v(\{1\})=\lim _{k \rightarrow+\infty} v([d-1 / k, d])=0,
$$

which is a contradiction with the fact that $v$ is not concentrated at 0 . Thus, we take $a \in(0, d)$ satisfying $v([a, d]) \neq 0$. Let $f \in W^{2,1}(0, a)$ be such that $f^{\prime}(0)=1$ and $f(a)=0$. Now for sufficiently large $k$, that is for $k$ satisfying $a+1 / k<d$, we define $g_{k} \in W^{2,1}(0, d)$ as $f$ on $(0, a)$, linear on $(a, a+1 / k)$ with $g_{k}(a)=0, g_{k}(a+1 / k)=1$, and equal to 1 on $(a+1 / k, d)$. By the Lebesgue dominated convergence theorem we have

$$
\lim _{k \rightarrow+\infty} \int_{a}^{d} g_{k} \mathrm{~d} v=v([a, d]) \neq 0,
$$

hence there is $k_{0}$ such that $\int_{a}^{d} g_{k_{0}} \mathrm{~d} v \neq 0$. If $F_{v} g_{k_{0}}=0$, then we are done, and otherwise we take

$$
g(x)= \begin{cases}f(x), & x \in[0, a), \\ -g_{k_{0}}(x) \frac{\int_{[0, a)} f \mathrm{~d} v}{\int_{[a, d]} g_{k_{0}} \mathrm{~d} v}, & x \in[a, d] .\end{cases}
$$

It follows that

$$
F_{\nu} g=\int_{[0, a)} f \mathrm{~d} v-\int_{[0, a)} f \mathrm{~d} v=0,
$$

and since $f(a)=g_{k_{0}}(a)=0$ we have $g \in W^{2,1}(0, d)$. Additionally $g^{\prime}(0)=f^{\prime}(0)=1$, and $g^{\prime}(d)=0$, because $g$ is constant in a neighbourhood of $d$.

Lemma 2.14 Let $J: W^{2,1}(S) \rightarrow W^{2,1}(S)$ be given by

$$
J \varphi:=\left(\left(F_{L, i} \varphi\right) h_{L, i}+\left(F_{R, i} \varphi\right) h_{R, i}\right)_{i \in \mathcal{N}}, \quad \varphi \in W^{2,1}(S),
$$

where $h_{L, i}$ and $h_{R, i}$ satisfy (2.35)-(2.36). Then for each $n \in \mathbb{N}$ the operator

$$
I_{n}:=I_{W^{2,1}(S)}-\kappa_{n}^{-1} J
$$

is an isomorphism of $W^{2,1}(S)$ with

$$
I_{n}^{-1}=I_{W^{2,1}(S)}+\kappa_{n}^{-1} J .
$$

Moreover

$$
\varphi \in D\left(A_{n}\right) \quad \text { if and only if } \quad I_{n} \varphi \in D\left(A_{0}\right) .
$$


Proof Fix $n \in \mathbb{N}$. By (2.35) it follows that $J^{2}=0$. Consequently

$$
J I_{n} \varphi=J \varphi
$$

for all $\varphi \in W^{2,1}(S)$. Hence if $I_{n} \varphi=0$, then $\varphi=\kappa^{-1} J \varphi=0$, proving that $I_{n}$ is one-toone. On the other hand, if $\varphi \in W^{2,1}(S)$, then

$$
I_{n}\left(\varphi+\kappa^{-1} J \varphi\right)=\varphi-\kappa^{-1} J \varphi+\kappa^{-1} J \varphi-\kappa^{-2} J^{2} \varphi=\varphi,
$$

which shows (2.38).

In order to check (2.39), assume first that $\varphi \in D\left(A_{n}\right)$. Then, by (2.36),

$$
(J \varphi)^{\prime}\left(L_{i}\right)=\left(F_{L, i} \varphi\right) h_{L, i}^{\prime}\left(L_{i}\right)+\left(F_{R, i} \varphi\right) h_{R, i}^{\prime}\left(L_{i}\right)=F_{L, i} \varphi, \quad i \in \mathcal{N} .
$$

Hence, using the definition of $I_{n}$ and the fact that $\varphi \in D\left(A_{n}\right)$ satisfies $\kappa_{n} L \varphi=F \varphi$, we obtain

$$
\left(I_{n} \varphi\right)^{\prime}\left(L_{i}\right)=\varphi^{\prime}\left(L_{i}\right)-\kappa_{n}^{-1} F_{L, i} \varphi=0, \quad i \in \mathcal{N} .
$$

Similarly $\left(I_{n} \varphi\right)^{\prime}\left(R_{i}\right)=0$ for all $i \in \mathcal{N}$, which means that $I_{n} \varphi \in D\left(A_{0}\right)$. Conversely, assume that $\psi:=I_{n} \varphi \in D\left(A_{0}\right)$. Then $\psi^{\prime}\left(L_{i}\right)=\psi^{\prime}\left(R_{i}\right)=0$. Consequently, calculating as in (2.41) and using (2.40), we have

$$
(J \psi)^{\prime}\left(L_{i}\right)=F_{L, i} \psi=F_{L, i} \varphi-\kappa_{n}^{-1} F_{L, i} J \varphi=F_{L, i} \varphi
$$

for all $i \in \mathcal{N}$. Therefore, by (2.38),

$$
\varphi^{\prime}\left(L_{i}\right)=\left(I_{n}^{-1} \psi\right)^{\prime}\left(L_{i}\right)=\psi^{\prime}\left(L_{i}\right)+\kappa_{n}^{-1} F_{L, i} \varphi=\kappa_{n}^{-1} F_{L, i} \varphi, \quad i \in \mathcal{N} .
$$

Similarly $\varphi^{\prime}\left(R_{i}\right)=\kappa_{n}^{-1} F_{R, i} \varphi$ for all $i \in \mathcal{N}$, which implies that $\varphi \in D\left(A_{n}\right)$, and completes the proof.

Lemma 2.14 says that $I_{n}$ is an isomorphism of $W^{2,1}(S)$ that transforms functions $\varphi$ 's satisfying $\kappa_{n} L \varphi=F \varphi$ to those satisfying homogeneous Neumann boundary conditions on each edge, that is, $L I_{n} \varphi=0$.

We denote by $A_{\text {ex }}$ the extended limit of $\left(A_{n}\right)_{n \in \mathbb{N}}$. Let also $K: W^{2,1}(S) \rightarrow W^{2,1}(S)$ be defined as $J$, see (2.37), with $h_{L, i}, h_{R, i}$ replaced by their second derivatives, that is,

$$
K \varphi:=\left(\left(F_{L, i} \varphi\right) h_{L, i}^{\prime \prime}+\left(F_{R, i} \varphi\right) h_{R, i}^{\prime \prime}\right)_{i \in \mathcal{N}^{\prime}}, \quad \varphi \in W^{2,1}(S)
$$

Lemma 2.15 The domain $D\left(A_{\mathrm{ex}}\right)$ contains $L_{0}^{1}(S)$, and

$$
(\varphi, \sigma K \varphi) \in A_{\mathrm{ex}}, \quad \varphi \in L_{0}^{1}(S) .
$$

Proof Fix $\varphi \in L_{0}^{1}(S)$ and set, see (2.38),

$$
\varphi_{n}:=I_{n}^{-1} \varphi=\varphi+\kappa_{n}^{-1} J \varphi .
$$


Since $\kappa_{n}^{-1} \rightarrow 0$ as $n \rightarrow+\infty$, the sequence $\left(\varphi_{n}\right)_{n \in \mathbb{N}}$ converges to $\varphi$ in $L^{1}(S)$ as $n \rightarrow+\infty$. What is more $\varphi_{n} \in D\left(A_{n}\right)$ for every $n \in \mathbb{N}$ by (2.39), and

$$
A_{n} \varphi_{n}=\kappa_{n} \sigma \varphi^{\prime \prime}+\sigma(J \varphi)^{\prime \prime}=\sigma K \varphi,
$$

where in the last equality we used the fact that $\varphi \in L_{0}^{1}(S)$ is constant on each edge, which guarantees that $\varphi^{\prime \prime}=0$. Hence $(\varphi, \sigma K \varphi) \in A_{\mathrm{ex}}$, and this completes the proof.

For the next lemma let $A_{\mathrm{ex}}^{0}$ be the extended limit of $\left(\kappa_{n}^{-1} A_{n}\right)_{n \in \mathbb{N}^{*}}$.

Lemma 2.16 For every $\varphi \in D\left(A_{0}\right)$ we have

$$
\left(\varphi, A_{0} \varphi\right) \in A_{\mathrm{ex}}^{0} .
$$

Proof Let $\varphi \in D\left(A_{0}\right)$ and set $\varphi_{n}:=I_{n}^{-1} \varphi$. Then $\varphi_{n} \in D\left(A_{n}\right)$ for all $n \in \mathbb{N}$ by (2.39). As in the previous lemma $\left(\varphi_{n}\right)_{n \in \mathbb{N}}$ converges to $\varphi$ in $L^{1}(S)$ as $n \rightarrow+\infty$, and

$$
\kappa_{n}^{-1} A_{n} \varphi_{n}=\sigma \varphi^{\prime \prime}+\kappa_{n}^{-1} \sigma(J \varphi)^{\prime \prime}=A_{0} \varphi+\kappa_{n}^{-1} \sigma K \varphi .
$$

Since $\lim _{n \rightarrow+\infty} \kappa_{n}^{-1}=0$ it follows that $\left(\varphi, A_{0} \varphi\right) \in A_{\mathrm{ex}}^{0}$, as claimed.

It follows by Proposition 2.4 that for the operator $A_{0}$ defined by (2.13), we have, see (2.30),

$$
\lim _{t \rightarrow+\infty} \mathrm{e}^{t A_{0}} \varphi=P \varphi
$$

for every $\varphi \in L^{1}(S)$. Furthermore, observe that for all $\varphi \in W^{2,1}(S)$, we have

$$
\begin{aligned}
P K \varphi & =\left(d_{i}^{-1} \int_{E_{i}}\left(\left(F_{L, i} \varphi\right) h_{L, i}^{\prime \prime}+\left(F_{R, i} \varphi\right) h_{R, i}^{\prime \prime}\right) \mathrm{d} m\right)_{i \in \mathcal{N}} \\
& =\left(d_{i}^{-1}\left(F_{R, i} \varphi-F_{L, i} \varphi\right)\right)_{i \in \mathcal{N}^{\prime}}
\end{aligned}
$$

where in the last equality we used (2.36). Therefore, see (2.32),

$$
Q \varphi=\sigma P K \varphi, \quad \varphi \in L_{0}^{1}(S) .
$$

Now we are ready to prove Theorem 2.11

Proof of Theorem 2.11 Fix $\omega$ as in Corollary 2.7, and let $\mathbb{X}:=L^{1}(S), \mathcal{A}_{n}:=A_{n}-\omega$, $\mathcal{A}:=\sigma K-\omega, \mathcal{A}_{0}:=A_{0}$, and $\varepsilon_{n}:=\kappa_{n}^{-1}$. Then $\mathcal{P}$ defined by (2.34) equals $P$. By Lemmas 2.15 and 2.16 conditions (i) and (ii) from Kurtz's theorem are satisfied. Moreover, $\mathcal{P} \mathcal{A}$ with domain $\mathbb{Y}=L_{0}^{1}(S)$ equals $Q-\omega$, since $P \varphi=\varphi$ for all $\varphi \in L_{0}^{1}(S)$. Therefore, by Theorem 2.12 ,

$$
\lim _{n \rightarrow+\infty} \mathrm{e}^{t\left(A_{n}-\omega\right)}=\mathrm{e}^{t(Q-\omega)} P
$$

strongly in $L^{1}(S)$, and the claim follows. 
Finally we restate Theorem 2.11 for the operators $A_{n}^{*}$ defined in Sect. 2.1. Denoting by $I_{i}^{E}$ the set of indices $j \neq i$ of edges incident to $E_{i}$, it follows by (2.3), that for every $\varphi=\left(\varphi_{i}\right)_{i \in \mathcal{N}} \in L_{0}^{1}(S)$ we have

$$
\begin{aligned}
Q \varphi & =\left(d_{i}^{-1} \sum_{j \in I_{i}^{E}}\left[\sigma_{j} l_{j i} \varphi\left(L_{j}\right)+\sigma_{j} r_{j i} \varphi\left(R_{j}\right)\right]-\sigma_{i} d_{i}^{-1}\left[l_{i} \varphi\left(L_{i}\right)+r_{i} \varphi\left(R_{i}\right)\right]\right)_{i \in \mathcal{N}} \\
& =\left(d_{i}^{-1} \sum_{j \neq i} \sigma_{j}\left(l_{j i}+r_{j i}\right) \varphi_{j}-\sigma_{i} d_{i}^{-1}\left(l_{i}+r_{i}\right) \varphi_{i}\right)_{i \in \mathcal{N}} .
\end{aligned}
$$

The isomorphic image of the operator $Q$ in $\mathbb{R}^{N}$ is given by

$$
\mathcal{I} Q \mathcal{I}^{-1}\left(\varphi_{i}\right)_{i=1, \ldots, N}, \quad\left(\varphi_{i}\right)_{i \in \mathcal{N}} \in \mathbb{R}^{N},
$$

where $\mathcal{I}$ is defined in (2.31). Therefore,

$$
\begin{aligned}
\mathcal{I} Q \mathcal{I}^{-1}\left(\varphi_{i}\right)_{i \in \mathcal{N}} & =\mathcal{I}\left(d_{i}^{-1} \sum_{j \neq i} \sigma_{j}\left(l_{j i}+r_{j i}\right) d_{j}^{-1} \varphi_{j}-\sigma_{i} d_{i}^{-1}\left(l_{i}+r_{i}\right) d_{i}^{-1} \varphi_{i}\right)_{i \in \mathcal{N}} \\
& =\left(\sum_{j \neq i} \sigma_{j} d_{j}^{-1}\left(l_{j i}+r_{j i}\right) \varphi_{j}-\sigma_{i} d_{i}^{-1}\left(l_{i}+r_{i}\right) \varphi_{i}\right)_{i \in \mathcal{N}}
\end{aligned}
$$

This shows that $Q$ may by identified with the matrix $\left(q_{i j}\right)_{i, j \in \mathcal{N}}$ given by

$$
q_{i j}:=\sigma_{j} d_{j}^{-1}\left(l_{j i}+r_{j i}\right), \quad i \neq j
$$

and

$$
q_{i i}:=-\sigma_{i} d_{i}^{-1}\left(l_{i}+r_{i}\right), \quad i \in \mathcal{N},
$$

in the sense that

$$
Q \varphi=\left(\varphi_{i}\right)_{i \in \mathcal{N}} \cdot\left(q_{i j}\right)_{i, j \in \mathcal{N}}, \quad \varphi=\left(\varphi_{i}\right)_{i \in \mathcal{N}} \in L_{0}^{1}(S)
$$

here $\left(\varphi_{i}\right)_{i \in \mathcal{N}}$ is treated as a row vector in $\mathbb{R}^{N}$. Notice that the matrix is the same as the matrix in Theorem 1.1. Therefore, as a corollary of Theorem 2.11 we obtain the following result.

Corollary 2.17 The conclusion of Theorem 2.11 holds with $A_{n}$ replaced by the operator $A_{n}^{*}$, see (2.4), and $Q$ given by (2.42).

\section{Analysis in $L^{2}(S)$}

The analysis of Sect. 2 was rather complicated in $L^{1}$ setting. Here we take the operator given by the same formula as $A_{n}$, but we change the space $L^{1}(S)$ to the Hilbert space $L^{2}(S)$. This allows us to use the machinery of sesquilinear forms, which 
simplifies the problem greatly. However, in terms of the underlying stochastic process, $L^{2}(S)$ is not a natural space to consider.

We set

$$
L^{2}(S):=\left\{u: u_{\mid E_{i}} \in L^{2}\left(E_{i}\right)\right\}
$$

where $L^{2}\left(E_{i}\right)$ is the complex Hilbert space of (equivalence classes of) Lebesgue square integrable complex functions on $E_{i}$, and the latter space is isometrically isomorphic to the standard $L^{2}\left(0, d_{i}\right)$ (see remarks at the beginning of Sect. 2.1). In contradistinction to $L^{1}(S)$, we denote elements of $L^{2}(S)$ by $u$ and $v$, instead of $\varphi$ and $\psi$. The space $L^{2}(S)$ equipped with the scalar product

$$
\langle u, v\rangle_{L^{2}(S)}:=\int_{S} u \bar{v} \mathrm{~d} m=\sum_{i \in \mathcal{N}} \int_{E_{i}} u_{i} \overline{v_{i}} \mathrm{~d} m=\sum_{i \in \mathcal{N}}\left\langle u_{i}, v_{i}\right\rangle_{L^{2}\left(E_{i}\right)}
$$

is a complex Hilbert space. By $H^{1}(S)$ we denote the Sobolev space $W^{1,2}(S) \subset L^{2}(S)$, that is $u \in H^{1}(S)$ if and only if $u \in L^{2}(S), u$ is weakly differentiable and $u^{\prime} \in L^{2}(S)$. Similarly we define $H^{2}(S)=W^{2,2}(S)$ as the space of $u \in L^{2}(S)$ such that $u$ and $u^{\prime}$ are weakly differentiable, and $u^{\prime}, u^{\prime \prime} \in L^{2}(S)$. We note that, in particular, $H^{1}(S)$ with the norm

$$
\|u\|_{H^{1}}:=\|u\|_{L^{2}(S)}+\left\|u^{\prime}\right\|_{L^{2}(S)}, \quad u \in H^{1}(S)
$$

is a Banach space, and by the Sobolev embedding theorem each $u \in H^{1}(S)$ has a continuous representant $\tilde{u}$ satisfying $\|\tilde{u}\|_{C(S)} \leq C\|u\|_{H^{1}(S)}$ for some $C>0$ that is independent of $u$.

For each $n \in \mathbb{N}$ we define the operator $A_{n}$ in $L^{2}(S)$ similarly as in Sect. 2.4, that is

$$
A_{n} u:=\kappa_{n} \sigma u^{\prime \prime}, \quad u \in D\left(A_{n}\right),
$$

where

$$
D\left(A_{n}\right):=\left\{u \in H^{2}(S): \kappa_{n} L u=F u\right\}
$$

with $L$ and $F$ defined as in (2.12), with $F_{L, i}$ and $F_{R, i}$ being functionals on $H^{1}(S)$. Provided that $F$ is bounded as an operator $H^{1}(S) \rightarrow \mathbb{R}^{2 N}$ we prove in Theorem 3.2 that $A_{n}$ 's generate holomorphic semigroups in $L^{2}(S)$ and, in Theorem 3.6, investigate their asymptotics.

\subsection{Sesquilinear forms}

In what follows we extensively use the theory of sesquilinear forms, see for example [16, Chapter 6] or [24, Chapter 1]. We recall that a sesquilinear form (or simply form $)$ in a complex Hilbert space $\left(H,\langle\cdot, \cdot\rangle_{H}\right)$ is a mapping $\mathfrak{a}: D(\mathfrak{a}) \times D(\mathfrak{a}) \rightarrow \mathbb{C}$ such that $\mathfrak{a}(\cdot, u)$ is linear and $\mathfrak{a}(u, \cdot)$ is antilinear for all $u \in D(\mathfrak{a})$. The set $D(\mathfrak{a})$ is a linear subspace of $H$ and is called the domain of $\mathfrak{a}$. We say that $\mathfrak{a}$ is densely defined if $D(\mathfrak{a})$ is a dense set in $H$, accretive if $\operatorname{Re} \mathfrak{a}(u, u) \geqslant 0$ for each $u \in D(\mathfrak{a})$, and closed if $D(\mathfrak{a})$ 
is a Hilbert space with respect to the inner product $\langle u, v\rangle_{\mathfrak{a}}:=\operatorname{Re} \mathfrak{a}(u, v)+\langle u, v\rangle_{H}$, $u, v \in D(\mathfrak{a})$. Moreover, we call $\mathfrak{a}$ sectorial if there exists $M>0$ such that

$$
|\operatorname{Ima}(u, u)| \leqslant M \operatorname{Re} \mathfrak{a}(u, u), \quad u \in D(\mathfrak{a}) .
$$

If $\left(\mathfrak{a}_{n}\right)_{n \in \mathbb{N}}$ is a sequence of forms in $H$, then we say that $\mathfrak{a}_{n}$ 's are uniformly sectorial if there exists $M>0$ (independent of $n$ ) such that (3.3) holds with a replaced by $\mathfrak{a}_{n}$ for $n \in \mathbb{N}$. Also, to shorten notation, we write $\mathfrak{a}(u)$ for $\mathfrak{a}(u, u)$.

For a densely defined form $\mathfrak{a}$ we define the associated operator $A$ in the following way. The domain $D(A)$ of $A$ is the set of $u \in D(\mathfrak{a})$ such that there exists $f \in D(\mathfrak{a})$ satisfying

$$
\mathfrak{a}(u, v)=-\langle f, v\rangle, \quad v \in D(\mathfrak{a}) .
$$

For $u \in D(\mathfrak{a})$ we set

$$
A u:=f .
$$

This definition is correct, since by the density of $D(\mathfrak{a})$ the element $f$ is unique. It turns out, see [16, Theorem VI.2.1] or [24, Theorem 1.52], that the operator associated with a densely defined, accretive, closed and sectorial form $\mathfrak{a}$ is the generator of a bounded holomorphic semigroup in $H$ denoted by $\left\{\mathrm{e}^{-t \mathfrak{a}}\right\}_{t \geqslant 0}$.

In order to state Ouhabaz's result (see [23, Theorem 5]), which is our main tool in this section, we need to introduce the notion of the degenerate semigroup related to a non densely defined form. Let $\mathfrak{a}$ be a form in $H$. If the domain $D(\mathfrak{a})$ is not dense in $H$, then there is no operator associated with the form $\mathfrak{a}$. However, we may consider the form in the closure $H_{0}$ of $D(\mathfrak{a})$ in $H$. Then $H_{0}$ is a Hilbert space and there is the operator $A_{H_{0}}$ associated with $\mathfrak{a}$ as restricted to $H_{0}$. If the form $\mathfrak{a}$ is accretive, closed and sectorial, then $A_{H_{0}}$ generates a bounded, holomorphic semigroup $\left\{\mathrm{e}^{t A_{H_{0}}}\right\}_{t \geqslant 0}$ in $H_{0}$. We extend this semigroup to the degenerate semigroup $\left\{\mathrm{e}^{-t \mathfrak{a}}\right\}_{t \geqslant 0}$ in $H$, by setting

$$
\mathrm{e}^{-t \mathfrak{a}} u:=\mathrm{e}^{t A_{H_{0}}} P_{H_{0}} u, \quad u \in H, t \geqslant 0,
$$

where $P_{H_{0}}$ is the orthogonal projection of $H$ onto $H_{0}$.

In our particular setup, we use the following special case of Ouhabaz's theorem (see [6, Theorem 3.2 and Corollary 3.3] for the general version).

Theorem 3.1 Let $\left(\mathfrak{a}_{n}\right)_{n \in \mathbb{N}}$ be a sequence of accretive, closed and uniformly sectorial forms defined on the same domain D in a Hilbert space H. Assume that

(i) $\operatorname{Rea}_{n}(u) \leqslant \operatorname{Rea}_{n+1}(u)$ for every $u \in D$,

(ii) for each $u \in D$ the imaginary part $\operatorname{Ima}_{n}(u)$ does not depend on $n \in \mathbb{N}$.

Then the form a defined by

$$
\mathfrak{a}(u, v):=\lim _{n \rightarrow+\infty} \mathfrak{a}_{n}(u, v), \quad u, v \in D(\mathfrak{a})
$$


with domain

$$
D(\mathfrak{a}):=\left\{u \in D: \sup _{n \in \mathbb{N}} \mathfrak{a}_{n}(u)<+\infty\right\},
$$

is accretive, closed and sectorial. Moreover, for every $u \in H$ and $t>0$,

$$
\lim _{n \rightarrow+\infty} \mathrm{e}^{-t \mathfrak{a}_{n}} u=\mathrm{e}^{-t \mathfrak{a}} u, \quad u \in H, t>0
$$

in $H$, and the convergence is uniform on compact subsets of $(0, \infty)$. If $u$ is in the closure of $D(\mathfrak{a})$, then (3.4) holds also for $t=0$, and the convergence is uniform on compact subsets of $[0,+\infty)$.

\subsection{Generation theorem in $L^{2}(S)$}

We prove a generation result analogous to Theorem 2.3.

Theorem 3.2 Assume that $F$ in (3.2) is bounded in the sense that

$$
\|F \varphi\|_{\mathbb{R}^{2 N}} \leq C_{F}\|\varphi\|_{H^{1}(S)}, \quad \varphi \in H^{1}(S)
$$

for some $C_{F}>0$. Then for each $n \in \mathbb{N}$ the operator $A_{n}$ given by (3.1) generates a holomorphic semigroup $\left\{\mathrm{e}^{t A_{n}}\right\}_{t \geqslant 0}$ in $L^{2}(S)$, and there exists $\gamma>0$ such that

$$
\left\|\mathrm{e}^{-\gamma t} \mathrm{e}^{t A_{n}}\right\|_{\mathcal{L}\left(L^{2}(S)\right)} \leqslant 1, \quad n \in \mathbb{N}, t \geqslant 0 .
$$

Throughout this section fix $n \in \mathbb{N}$. We begin by finding a form $\mathfrak{a}_{n}$ in $L^{2}(S)$ such that $A_{n}$ is the operator associated with $\mathfrak{a}_{n}$. Define the form $\mathfrak{b}_{n}$ in $L^{2}(S)$ by

$$
\mathfrak{b}_{n}(u, v):=\kappa_{n}\left\langle\sigma u^{\prime}, v^{\prime}\right\rangle_{L^{2}(S)}
$$

with the domain $D\left(\mathfrak{b}_{n}\right):=H^{1}(S)$. Let $u \in D\left(A_{n}\right)$ and $v \in H^{1}(S)$. Integration by parts gives

$$
\int_{E_{i}} u^{\prime \prime} \bar{v} \mathrm{~d} m=-\int_{E_{i}} u^{\prime} \bar{v}^{\prime} \mathrm{d} m+u^{\prime}\left(R_{i}\right) \bar{v}\left(R_{i}\right)-u^{\prime}\left(L_{i}\right) \bar{v}\left(L_{i}\right), \quad i \in \mathcal{N} .
$$

Hence, since $\kappa_{n} L u=F u$,

$$
\left\langle A_{n} u, v\right\rangle_{L^{2}(S)}=-\mathfrak{b}_{n}(u, v)-\mathfrak{c}(u, v),
$$

where $\mathrm{c}$ is the form in $L^{2}(S)$ given by

$$
\mathfrak{c}(u, v):=\sum_{i \in \mathcal{N}} \sigma_{i}\left[\left(F_{L, i} u\right) \bar{v}\left(L_{i}\right)-\left(F_{R, i} u\right) \bar{v}\left(R_{i}\right)\right]
$$

with domain $D(\mathfrak{c}):=H^{1}(S)$. Note that $c$ does not depend on $n$. Formula (3.7) suggests that we should set $D\left(\mathfrak{a}_{n}\right):=H^{1}(S)$ and define 


$$
\mathfrak{a}_{n}:=\mathfrak{b}_{n}+\mathfrak{c} .
$$

The space $H^{1}(S)$ is dense in $L^{2}(S)$, therefore, in order to prove that the operator associated with $\mathfrak{a}_{n}$ generates a holomorphic semigroup, we are left with proving that the form $\mathfrak{a}_{n}$ is accretive, closed and sectorial.

For the proofs of Lemma 3.3 and Proposition 3.4 it is useful to denote

$$
\sigma_{\min }:=\min _{i \in \mathcal{N}} \sigma_{i}, \quad \sigma_{\max }:=\max _{i \in \mathcal{N}} \sigma_{i} .
$$

Lemma 3.3 The form $\mathfrak{b}_{n}$ is accretive and closed.

Proof For $\sqrt{\sigma}:=\left(\sqrt{\sigma_{i}}\right)_{i \in \mathcal{N}}$ we have

$$
\mathfrak{b}_{n}(u)=\kappa_{n}\left\|\sqrt{\sigma} u^{\prime}\right\|_{L^{2}(S)}^{2}, \quad u \in H^{1}(S),
$$

which proves accretivity. Observe that

$$
\kappa_{n} \sigma_{\min }\left\|u^{\prime}\right\|_{L^{2}(S)}^{2} \leqslant \mathfrak{b}_{n}(u) \leqslant \kappa_{n} \sigma_{\max }\left\|u^{\prime}\right\|_{L^{2}(S)}, \quad u \in H^{1}(S) .
$$

Hence the norm $\|\cdot\|_{\mathfrak{b}_{n}}$ associated with $\mathfrak{b}_{n}$ is equivalent to the standard norm in $H^{1}(S)$ (which is a Hilbert space), and the claim follows.

Proposition 3.4 The form $\mathfrak{a}_{n}$ is closed and there exists $\gamma>0$ such that the form $\mathfrak{a}_{n}+\gamma$ is sectorial with

$$
\left|\operatorname{Im}\left(\mathfrak{a}_{n}+\gamma\right)(u)\right| \leqslant \operatorname{Re}\left(\mathfrak{a}_{n}+\gamma\right)(u), \quad u \in H^{1}(S) .
$$

Here, by $\mathfrak{a}_{n}+\gamma$ we mean the form defined by $\left(\mathfrak{a}_{n}+\gamma\right)(u, v)=\mathfrak{a}_{n}(u, v)+\gamma\langle u, v\rangle_{L^{2}(S)}$.

Proof By (3.5) it follows that for some $c>0$ we have

$$
|\mathfrak{c}(u)| \leqslant c\|u\|_{H^{1}(S)}\|u\|_{L^{\infty}(S)}, \quad u \in H^{1}(S),
$$

where $\|\cdot\|_{L^{\infty}(S)}$ is the essential supremum norm. By the Gagliardo-Nirenberg interpolation (see [12, Theorem 12.83]), there exists $C>0$ such that

$$
\|u\|_{L^{\infty}(S)}^{2} \leqslant C\|u\|_{L^{2}(S)}\left\|u^{\prime}\right\|_{L^{2}(S)}, \quad u \in H^{1}(S),
$$

hence,

$$
|\mathfrak{c}(u)| \leq c C\left[\|u\|_{L^{2}(S)}^{3 / 2}\left\|u^{\prime}\right\|_{L^{2}(S)}^{1 / 2}+\|u\|_{L^{2}(S)}^{1 / 2}\left\|u^{\prime}\right\|_{L^{2}(S)}^{3 / 2}\right], \quad u \in H^{1}(S) .
$$

Using Young's inequality, for all $a, b \geq 0$ and all $\varepsilon>0$ we have 


$$
a^{3 / 2} b^{1 / 2} \leq \frac{3 a^{2}}{4 \varepsilon}+\frac{\varepsilon^{3} b^{2}}{4} .
$$

Therefore, there exists $\gamma>0$ such that

$$
|\mathfrak{c}(u)| \leqslant \frac{\kappa_{n} \sigma_{\min }}{2}\left\|u^{\prime}\right\|_{L^{2}(S)}^{2}+\frac{\gamma}{2}\|u\|_{L^{2}(S)}^{2}, \quad u \in H^{1}(S) .
$$

Therefore, see (3.9),

$$
|\mathfrak{c}(u)| \leqslant \frac{1}{2} \mathfrak{b}_{n}(u)+\frac{\gamma}{2}\|u\|_{L^{2}(S)}^{2}, \quad u \in H^{1}(S) .
$$

This means that $\mathfrak{c}$ is $\mathfrak{b}_{n}$-bounded with $\mathfrak{b}_{n}$-bound $1 / 2$ (see [24, Definition 1.17]). Using [16, Theorem VI.3.4] or [24, Theorem 1.19], it follows that the form $\mathfrak{a}_{n}=\mathfrak{b}_{n}+\mathfrak{c}$ is closed as a relatively bounded perturbation of the closed form $\mathfrak{b}_{n}$.

To show the second part of the proposition notice that by (3.8), (3.9), and (3.11) we have

$$
\left|\operatorname{Ima}_{n}(u)\right|=|\operatorname{Imc}(u)| \leqslant \frac{1}{2} \mathfrak{b}_{n}(u)+\frac{\gamma}{2}\|u\|_{L^{2}(S)}^{2},
$$

and

$$
\operatorname{Re}_{n}(u) \geqslant \mathfrak{b}_{n}(u)-|\operatorname{Rec}(u)| \geqslant \frac{1}{2} \mathfrak{b}_{n}(u)-\frac{\gamma}{2}\|u\|_{L^{2}(S)}^{2}
$$

for all $u \in H^{1}(S)$. Combining these two inequalities we obtain

$$
\left|\operatorname{Im}\left(\mathfrak{a}_{n}+\gamma\right)(u)\right|=\left|\operatorname{Ima}_{n}(u)\right| \leqslant \operatorname{Re}_{n}(u)+\gamma\|u\|_{L^{2}(S)}^{2},
$$

which proves (3.10).

Proposition 3.5 The operator associated with $\mathfrak{a}_{n}$ is $A_{n}$.

Proof Let $B_{n}$ be the operator associated with $\mathfrak{a}_{n}$. We claim that $B_{n}$ is exactly $A_{n}$. Formula (3.7) shows that $D\left(A_{n}\right) \subseteq D\left(B_{n}\right)$ and $B_{n} u=A_{n} u$ for $u \in D\left(A_{n}\right)$. On the other hand let $u \in D\left(B_{n}\right)$. There exists $f \in H^{1}(S)$ such that $\mathfrak{a}_{n}(u, v)=-\langle f, v\rangle_{L^{2}(S)}$ for all $v \in H^{1}(S)$. Choose $v \in H^{1}(S)$ that on each edge is a compactly supported smooth function. Then $c(u, v)=0$ and consequently

$$
\mathfrak{a}_{n}(u, v)=\mathfrak{b}_{n}(u, v)=\kappa_{n}\left\langle\sigma u^{\prime}, v^{\prime}\right\rangle_{L^{2}(S)} .
$$

Therefore $\langle f, v\rangle_{L^{2}(S)}=-\kappa_{n}\left\langle\sigma u^{\prime}, v^{\prime}\right\rangle_{L^{2}(S)}$, which proves that $u \in H^{2}(S)$ and $f=B_{n} u=\kappa_{n} \sigma u^{\prime \prime}$. Now for fixed $i \in \mathcal{N}$ choose $v \in H^{1}(S)$ with

$$
v\left(R_{i}\right) \neq 0, \quad v\left(L_{i}\right)=0, \quad \text { and } \quad v\left(R_{j}\right)=v\left(L_{j}\right)=0, \quad j \in \mathcal{N}, j \neq i .
$$

Then 


$$
\mathfrak{c}(u, v)=-\sigma_{i}\left(F_{R, i} u\right) \bar{v}\left(R_{i}\right)
$$

and, integrating by parts,

$$
\mathfrak{b}_{n}(u, v)=-\kappa_{n}\left\langle\sigma u^{\prime \prime}, v\right\rangle_{L^{2}(S)}+\kappa_{n} \sigma_{i} u^{\prime}\left(R_{i}\right) \bar{v}\left(R_{i}\right) .
$$

Hence

$$
\mathfrak{a}_{n}(u, v)=-\kappa_{n}\left\langle\sigma u^{\prime \prime}, v\right\rangle_{L^{2}(S)}+\sigma_{i}\left[\kappa_{n} u^{\prime}\left(R_{i}\right)-F_{R, i} u\right] \bar{v}\left(R_{i}\right) .
$$

This equality, since $\mathfrak{a}_{n}(u, v)=-\kappa_{n}\left\langle\sigma u^{\prime \prime}, v\right\rangle$, is equivalent to

$$
\kappa_{n} u^{\prime}\left(R_{i}\right)=F_{R, i} u \text {. }
$$

In the same way we prove that

$$
\kappa_{n} u^{\prime}\left(L_{i}\right)=F_{L, i} u \text {. }
$$

This means that $\kappa_{n} L u=F u$ and, since $u \in H^{2}(S)$, it follows that $u \in D\left(A_{n}\right)$. Finally $D\left(B_{n}\right)=D\left(A_{n}\right)$ and $B_{n} u=A_{n} u$ for all $u \in D\left(A_{n}\right)$.

Proof of Theorem 3.2 Let $\gamma>0$ be as in Proposition 3.4. Then the form $\mathfrak{a}_{n}+\gamma$ is densely defined, accretive, closed and sectorial. Moreover, by Proposition 3.5, the operator associated with $\mathfrak{a}_{n}+\gamma$ is $A_{n}-\gamma$. Therefore, by [24, Theorem 1.52], it follows that $A_{n}-\gamma$ generates a holomorphic contraction semigroup in $L^{2}(S)$. Hence, $A_{n}$ generates a holomorphic semigroup in $L^{2}(S)$, and since

$$
\left\|\mathrm{e}^{-\gamma} \mathrm{e}^{t A_{n}}\right\|_{\mathcal{L}\left(L^{2}(S)\right)} \leqslant 1, \quad t \geqslant 0, n \in \mathbb{N}
$$

inequality (3.6) holds.

\subsection{Convergence result in $L^{2}(S)$}

Let $L_{0}^{2}(S)$ be the closed subspace of $L^{2}(S)$ consisting of complex functions that are constant on each edge. Similarly as for $L_{0}^{1}(S)$ defined in Sect. 2.4, the space $L_{0}^{2}(S)$ is isometrically isomorphic to $\mathbb{C}^{N}$ equipped with the appropriate scalar product.

Let $P$ be the projection of $L^{2}(S)$ onto $L_{0}^{2}(S)$ given by (2.30), and let $Q$ be the operator in $L_{0}^{2}(S)$ defined as in $L_{0}^{1}(S)$ by formula (2.32). That is

$$
P u:=\left(d_{i}^{-1} \int_{E_{i}} u \mathrm{~d} m\right)_{i \in \mathcal{N}}, \quad Q u:=\left(d_{i}^{-1}\left(F_{R, i} u-F_{L, i} u\right)\right)_{i \in \mathcal{N}}
$$

for all $u$ in $L^{2}(S)$ and $L_{0}^{2}(S)$, respectively. We stress that, in contradistinction to Sect. 2.4, here we do not need to assume that $F=\left(F_{L, i}, F_{R, i}\right)$ is of the form (2.28), and we prove Theorem 3.6 for $F$ that is merely bounded in $H^{1}(S)$. 
Theorem 3.6 Assume that (3.5) holds. Then, for the generators $A_{n}$ 's defined by (3.1), we have

$$
\lim _{n \rightarrow+\infty} \mathrm{e}^{t A_{n}} u=\mathrm{e}^{t Q} P u, \quad u \in L^{2}(S), t>0
$$

in $L^{2}(S)$, where $P$ and $Q$ are given by (3.12). The convergence is uniform on compact subsets of $(0,+\infty)$. If $u \in L_{0}^{2}(S)$, then (3.13) holds also for $t=0$, and the convergence if uniform on compact subsets of $[0,+\infty)$.

For $n \in \mathbb{N}$ let $\mathfrak{a}_{n}$ be the form in $L^{2}(S)$ defined by (3.8). Fix $\gamma>0$ as in Proposition 3.4 and define

$$
\mathfrak{a}_{n}^{\gamma}:=\mathfrak{a}_{n}+\gamma=\mathfrak{b}_{n}+\mathfrak{c}+\gamma, \quad n \in \mathbb{N}
$$

with the domain

$$
D\left(\mathfrak{a}_{n}^{\gamma}\right):=D\left(\mathfrak{a}_{n}\right)=H^{1}(S)
$$

Lemma 3.7 The sequence $\left(\mathfrak{a}_{n}^{\gamma}\right)_{n \in \mathbb{N}}$ consists of accretive, closed and uniformly sectorial forms. Moreover,

$$
\operatorname{Re}_{n}^{\gamma}(u) \leqslant \operatorname{Re}_{n+1}^{\gamma}(u)
$$

and

$$
\operatorname{Ima}_{n}^{\gamma}(u)=\operatorname{Imc}(u)
$$

for all $n \in \mathbb{N}, u \in H^{1}(S)$.

Proof The first part is a consequence of Proposition 3.4. For the second observe that

$$
\mathfrak{a}_{n}^{\gamma}(u)=\mathfrak{b}_{n}(u)+\mathfrak{c}(u)+\gamma\|u\|_{L^{2}(S)}^{2}, \quad n \in \mathbb{N}, u \in H^{1}(S) .
$$

The claim follows from the fact that $\kappa_{n} \leqslant \kappa_{n+1}$ and $\mathfrak{b}_{n}(u)=\operatorname{Re} \mathfrak{b}_{n}(u)$ for all $n \in \mathbb{N}$ and $u \in H^{1}(S)$.

Let $\mathfrak{a}^{\gamma}$ be the form in $L^{2}(S)$ defined by

$$
\mathfrak{a}^{\gamma}(u, v):=\lim _{n \rightarrow+\infty} \mathfrak{a}_{n}^{\gamma}(u, v), \quad u, v \in D\left(\mathfrak{a}^{\gamma}\right)
$$

with domain

$$
D\left(\mathfrak{a}^{\gamma}\right):=\left\{u \in H^{1}(S): \sup _{n \in \mathbb{N}} \mathfrak{a}_{n}^{\gamma}(u)<+\infty\right\}
$$

This definition makes sense because the limit of $\mathfrak{a}_{n}^{\gamma}(u)$ as $n \rightarrow+\infty$ exists, and we may define $\mathfrak{a}^{\gamma}$ by the polarization equality.

Lemma 3.8 We have 


$$
D\left(\mathfrak{a}^{\gamma}\right)=L_{0}^{2}(S)
$$

and

$$
\mathfrak{a}^{\gamma}(u, v)=\mathfrak{c}(u, v)+\gamma\langle u, v\rangle_{L^{2}(S)}, \quad u, v \in L_{0}^{2}(S) .
$$

Proof Let $u \in H^{1}(S)$ and observe that $u \in D\left(\mathfrak{a}^{\gamma}\right)$ if and only if

$$
\sup _{n \in \mathbb{N}} \mathfrak{b}_{n}(u)<+\infty \text {. }
$$

By (3.9), the last condition holds if and only if $u^{\prime}=0$ in $L^{2}(S)$, since $\kappa_{n} \rightarrow+\infty$ as $n \rightarrow+\infty$. This completes the proof, because $u^{\prime}=0$ is equivalent to $u \in L_{0}^{2}(S)$, and the formula (3.16) follows now immediately from (3.15) and (3.14).

Let $\mathfrak{c}_{0}$ be the restriction of $\mathfrak{c}$ to $L_{0}^{2}(S)$, that is the form in $L_{0}^{2}(S)$ given by

$$
\mathfrak{c}_{0}(u, v):=\mathfrak{c}(u, v), \quad u, v \in L_{0}^{2}(S) .
$$

Lemma 3.9 The operator associated with $\mathfrak{c}_{0}$ is $Q$ given by (3.12).

Proof Let $u, v \in L_{0}^{2}(S)$. Therefore, see (2.42),

$$
\mathfrak{c}_{0}(u, v)=\sum_{i \in \mathcal{N}} \sigma_{i} d_{i}^{-1}\left\langle F_{R, i} u-F_{L, i} u, v\right\rangle_{L^{2}\left(E_{i}\right)}=-\langle Q u, v\rangle_{L^{2}(S)},
$$

and the claim follows.

Corollary 3.10 The operator associated with $\mathfrak{a}^{\gamma}$, as a form in $L_{0}^{2}(S)$, is $Q-\gamma$, where $Q$ is given by (3.12).

Proof The claim is a consequence of (3.16), Lemmas 3.8, and 3.9.

Finally, we are ready to prove our convergence result in $L^{2}(S)$.

Proof of Theorem 3.6 By Lemma 3.7 the assumptions of Theorem 3.1 hold for the sequence $\left(\mathfrak{a}_{n}^{\gamma}\right)_{n \in \mathbb{N}}$. Hence,

$$
\lim _{n \rightarrow+\infty} \mathrm{e}^{-t \mathfrak{a}_{n}^{\gamma}} u=\mathrm{e}^{-t \mathbf{a}^{\gamma}} u, \quad u \in L^{2}(S) .
$$

By Proposition 3.5, $A_{n}-\gamma$ is associated with $\mathfrak{a}_{n}^{\gamma}$, and hence by Corollary 3.10 we can rewrite the above relation in the form

$$
\lim _{n \rightarrow+\infty} \mathrm{e}^{t\left(A_{n}-\gamma\right)} u=\mathrm{e}^{t(Q-\gamma)} P u, \quad u \in L^{2}(S),
$$

which is equivalent to (3.13). 
Acknowledgements I would like to thank an anonymous referee for valuable comments which helped to improve the paper.

Open Access This article is licensed under a Creative Commons Attribution 4.0 International License, which permits use, sharing, adaptation, distribution and reproduction in any medium or format, as long as you give appropriate credit to the original author(s) and the source, provide a link to the Creative Commons licence, and indicate if changes were made. The images or other third party material in this article are included in the article's Creative Commons licence, unless indicated otherwise in a credit line to the material. If material is not included in the article's Creative Commons licence and your intended use is not permitted by statutory regulation or exceeds the permitted use, you will need to obtain permission directly from the copyright holder. To view a copy of this licence, visit http://creativecommons.org/licen ses/by/4.0/.

\section{References}

1. Arendt, W., Batty, C., Hieber, M., Neubrander, F.: Vector-Valued Laplace Transforms and Cauchy Problems. Birkhäuser, Basel (2011). https://doi.org/10.1007/978-3-0348-0087-7

2. Banasiak, J., Falkiewicz, A., Namayanja, P.: Asymptotic state lumping in transport and diffusion problems on networks with applications to population problems. Math. Models Methods Appl. Sci. 26(2), 215-247 (2016). https://doi.org/10.1142/S0218202516400017

3. Banasiak, J., Falkiewicz, A., Namayanja, P.: Semigroup approach to diffusion and transport problems on networks. Semigroup Forum 93(3), 427-443 (2016). https://doi.org/10.1007/s0023 3-015-9730-4

4. Bobrowski, A.: From diffusions on graphs to Markov chains via asymptotic state lumping. Ann. Henri Poincaré 13(6), 1501-1510 (2012). https://doi.org/10.1007/s00023-012-0158-z

5. Bobrowski, A.: Convergence of One-parameter Operator Semigroups. Cambridge University Press, Cambridge (2016). https://doi.org/10.1017/CBO9781316480663

6. Bobrowski, A., Kaźmierczak, B., Kunze, M.: An averaging principle for fast diffusions in domains separated by semi-permeable membranes. Math. Models Methods Appl. Sci. 27(4), 663-706 (2017). https://doi.org/10.1142/S0218202517500130

7. Bobrowski, A., Morawska, K.: From a PDE model to an ODE model of dynamics of synaptic depression. Discrete Cont. Dyn. Syst. B 17(6), 2313-2327 (2012). https://doi.org/10.3934/dcdsb .2012.17.2313

8. Engel, K.J., Kramar Fijavž, M.: Waves and diffusion on metric graphs with general vertex conditions. Evolut. Equ. Control Theory 8(3), 633-661 (2019). https://doi.org/10.3934/eect.2019030

9. Engel, K.J., Nagel, R.: One-parameter Semigroups for Linear Evolution Equations. Springer, New York (2000). https://doi.org/10.1007/b97696

10. Ethier, S.N., Kurtz, T.G.: Markov processes. In: Ethier, S.N., Kurtz, T.G. (eds.) Characterization and Convergence. Wiley, New York (1986). https://doi.org/10.1002/9780470316658

11. Folland, G.: Real Analysis. Wiley, New York (1999)

12. Giovanni, L.: A First Course in Sobolev Spaces. American Mathematical Society, Providence (2017)

13. Gregosiewicz, A.: Asymptotic behaviour of diffusion on graphs. In: Banek, T., Kozłowski, E. (eds.) Probability in Action, vol. 1, pp. 83-96. Lublin University of Technology Press, Lublin (2014)

14. Greiner, G.: Perturbing the boundary conditions of a generator. Houston J. Math. 13(2), 213-229 (1987)

15. Gwiżdż, P., Tyran-Kamińska, M.: Positive semigroups and perturbations of boundary conditions. Positivity (2019). https://doi.org/10.1007/s11117-019-00644-w

16. Kato, T.: Perturbation Theory for Linear Operators. Springer, Berlin (1995). https://doi. org/10.1007/978-3-642-66282-9

17. Lasota, A., Mackey, M.: Chaos, Fractals, and Noise. Springer, New York (1994). https://doi. org/10.1007/978-1-4612-4286-4

18. Liggett, T.: Continuous Time Markov Processes. American Mathematical Society, Providence (2010). https://doi.org/10.1090/gsm/113 
19. Lorenzi, L., Lunardi, A., Metafune, G., Pallara, D.: Analytic Semigroups and Reaction-Diffusion Problems (2005). https://www.math.kit.edu/iana3/ schnaubelt/media/isem04.pdf. Internet Seminar 2004-2005

20. Mugnolo, D.: Semigroup Methods for Evolution Equations on Networks. Springer, Cham (2014). https://doi.org/10.1007/978-3-319-04621-1

21. Nagel, R. (ed.): One-parameter Semigroups of Positive Operators. Springer, Cham (1986)

22. Nickel, G.: A new look at boundary perturbations of generators. Electron. J. Differ. Equ. 95, 14 (2004)

23. Ouhabaz, E.M.: Second order elliptic operators with essential spectrum $[0, \infty)$ on $L^{p}$. Commun. Partial Differ. Equ. 20(5-6), 763-773 (1995). https://doi.org/10.1080/03605309508821114

24. Ouhabaz, E.M.: Analysis of Heat Equations on Domains. Princeton University Press, Princeton (2005)

25. Pazy, A.: Semigroups of Linear Operators and Applications to Partial Differential Equations. Springer, New York (1983). https://doi.org/10.1007/978-1-4612-5561-1

26. Rudnicki, R., Tyran-Kamińska, M.: Piecewise Deterministic Markov Processes in Biological Models, vol. 113. Springer, Cham (2015). https://doi.org/10.1007/978-3-319-12145-1_15

27. Simon, B.: A canonical decomposition for quadratic forms with applications to monotone convergence theorems. J. Funct. Anal. 28(3), 377-385 (1978). https://doi.org/10.1016/00221236(78)90094-0

Publisher's Note Springer Nature remains neutral with regard to jurisdictional claims in published maps and institutional affiliations. 